\title{
A Nonlocal Strain Gradient Approach for Out-of-Plane Vibration of Axially Moving Functionally Graded Nanoplates in a Hygrothermal Environment
}

\author{
Chengxiu Zhu, ${ }^{1}$ Jianwei Yan, ${ }^{2}$ Pingyuan Wang, ${ }^{1}$ and Cheng $\operatorname{Li} \mathbb{D}^{1,3}$ \\ ${ }^{1}$ School of Rail Transportation, Soochow University, Suzhou 215131, China \\ ${ }^{2}$ Engineering Research \& Development Centre for Underground Technology of Jiangxi Province, School of Civil Engineering \\ and Architecture, East China Jiaotong University, Nanchang, Jiangxi 330013, China \\ ${ }^{3}$ Guangxi Key Laboratory of Cryptography and Information Security, Guilin University of Electronic Technology, \\ Guilin 541004, China \\ Correspondence should be addressed to Cheng Li; licheng@suda.edu.cn
}

Received 22 July 2021; Accepted 3 November 2021; Published 19 November 2021

Academic Editor: Fabio Botta

Copyright (C) 2021 Chengxiu Zhu et al. This is an open access article distributed under the Creative Commons Attribution License, which permits unrestricted use, distribution, and reproduction in any medium, provided the original work is properly cited.

\begin{abstract}
Vibration analyses on axially moving functionally graded nanoplates exposed to hygrothermal environments are presented. The theoretical model of the nanoplate is described via the Kirchhoff plate theory in conjunction with the concept of the physical neutral layer. By employing the nonlocal strain gradient theory, the governing equation of motion is derived based on Hamilton's principle. The composite beam function method, as well as the complex modal approach, is utilized to obtain the vibration frequencies of axially moving functionally graded nanoplates. Some benchmark results related to the effects of temperature changing, moisture concentration, axial speed, aspect ratio, nonlocal parameter, and the material characteristic scale parameter on the stiffness of axially moving functionally graded nanoplates are obtained. The results reveal that with increasing the nonlocal parameter, gradient index, temperature changing, moisture concentration, and axial speed, the vibration frequencies decrease. The frequencies increase while increasing the material characteristic scale parameter and aspect ratio. Moreover, there is an interaction between the nonlocal parameter and material characteristic scale parameter, influencing and restricting each other.
\end{abstract}

\section{Introduction}

With the rise of micro/nanotechnologies, the development and application of micro/nano-electromechanical systems (MEMS/NEMS) have developed rapidly, such as $\mathrm{micro} /$ nanosensors, micro/nanoresonators, smart wearable devices, and medical nanorobots $[1,2]$. The systematic and in-depth understandings of the mechanical properties of these devices and their key micro/nanocomponents are needed to provide theoretical guidance for the mechanical-based design and regulation $[3,4]$. So far, there have been numerous investigations on this topic, especially the studies on the mechanical behavior of uniform nanomaterials and structures based on the molecular dynamic simulation or nonclassical continuum approaches [5-9]. These results play an important role in perfecting performances and promoting the industrialization of MEMS/NEMS [10] and cellular mechanics [11]. However, while the MEMS/NEMS work in some harsh environments, such as high temperature, high pressure, humidity and heat, high-strain rate, and highspeed impact, the nanostructure composed of a single uniform material gradually fails to meet the engineering requirements [12]. Introducing the concept of functionally graded materials at macroscale into micro/ nanoscale and constructing functionally graded nanostructures is one of the effective ways to overcome the problems in extreme working environments [13-15]. Functionally graded nanostructures, as one of the components in nanodevices or nanosystems, may produce an unwanted vibration caused by external excitations or their own factors during work. Hence, the studies on the 
vibration characteristics of functionally graded nanostructures are of great significance.

To study the dynamic behaviors of micro/nanodevices composed of functionally graded nanostructures, there are generally three kinds of theoretical approaches including the discrete methods [16], modified continuum theories [17], and atom-continuum multiscale models [18]. The discrete methods refer to that the micro/nanomaterials and structures are equivalent to a number of molecules or atoms, and the dynamics of materials and structures can be simulated by calculating the interaction between molecules or atoms. For a special micro/nanosystem with a large number of molecules/atoms, the application of discrete methods may take plenty of time with a higher requirement of computing hardware. Therefore, the modified continuum theories that regard the nanomaterials and structures as generalized continuous structures are more suitable for this case. Using the traditional analysis thought and modeling procedure (e.g., Hamilton's principle) of the macroscopic continuum, the mechanical properties of nanomaterials and structures can be predicted by establishing the nonclassical continuum constitutive relations containing internal characteristic scale parameters of the nanomaterials. Although the modified continuum theories still adopt the basic ideas of a classical macroscopic continuum, the constitutive relations of macroscopic continuum mechanics have not been duplicated directly but improve them to include new phenomena at the nanoscale, such as a long-range interaction between atoms and strains with higher-order gradient. The atom-continuum multiscale models aim to establish an atomic calculation framework on the micro/nanoscale and a continuum framework on the macroscale according to different geometric sizes in different dimensions of micro/nanomaterials and structures, so as to develop the spatial multiscale model. It may also introduce temporal multiscale into the dynamic analysis of atom-continuum multiscale models. In the present study, the dynamics of two-dimensional functionally graded nanostructures are investigated by using the modified continuum theories. Hence, the nonclassical continuum model is applied to characterize the inherent features at the nanoscale. Since functionally graded nanostructures are composed of inhomogeneous materials and there are a huge number of molecules/atoms contained in two-dimensional functionally graded nanostructures, it is relatively difficult to deal with inhomogeneous materials and structures by molecular dynamic simulation, atomic simulation, or atomcontinuum multiscale models.

The modified continuum theories mainly include the micropolar and micromorphic theory [19], the couple stress theory [20], the strain gradient theory [21], and the nonlocal theory [22], which have been widely applied in the mechanical analysis of micro/nano-structures. For example, Li et al. explored the size dependence of nanorods [23] and nanobeams [24] based on the nonlocal integral model, and the results show that the nonlocal effect was more sensitive in the thickness direction, which implies that the thickness effect plays a dominant role in the contribution of size dependence. Jalaei and Civalek [25] investigated the dynamic instability of viscoelastic porous functionally graded nanobeams embedded on the visco-Pasternak medium according to Eringen's differential law, and the model is exposed to the magnetic field as well as an axial oscillating load. Lu et al. [26] performed the factors influencing the postbuckling properties of multilayer microtubules reinforced by functionally graded graphene platelets containing initial geometrical imperfection via the modified couple stress theory.

In recent years, with the continuous deepening of the research, these modified continuum theories have shown some disadvantages [27]. For example, the internal characteristic scale parameters of the same nanomaterial are different in different studies due to the inconsistency in the determination methods. For another example, there have been different kinds of nonlocal effects predicted. The equivalent stiffness softening and stiffness hardening models were established, and even some paradoxes were found in the literature. On the basis of these theoretical models, Lim et al. [28] proposed a nonlocal strain gradient theory. By introducing three internal characteristic scale parameters, the nonlocal effects of classical strain and strain gradients, as well as the gradient effects of traditional Eringen's nonlocal stress and high-order nonlocal stresses are comprehensively considered in the theory. Therefore, the theoretical predictions by the nonlocal strain gradient theory were satisfactorily consistent with the experimental results [29]. Recently, the nonlocal strain gradient theory has become one of the most widely applied nonclassical continuum approaches [30]. For instance, Saffaria et al. [31] studied the dynamics of a cantilever nanotube resting on a nonlinear viscous Pasternak foundation in the context of the nonlocal strain gradient theory, in which the viscoelastic fluid was conveyed, and the magnetic field and harmonic loads were also taken into account. Based on the nonlocal strain gradient theory, Li et al. [32] examined the effect of size-dependent parameters on the stiffness of functionally gradient beams using the Timoshenko beam model. To sum up, the strain gradient theory is applied to analyze the free vibration of functionally graded nanoplates so as to obtain accurate mechanical results.

Generally, the functionally graded nanostructures can be modeled as one-dimensional beam/tube/rod structures, two-dimensional plate/shell/film structures, and three-dimensional solid structures according to their size characteristics. Among them, the one-dimensional functionally graded structures have been fully studied [33]. In terms of functionally graded nanoplates plate/shell/film, there is also some research literature. For example, Lu et al. [34] developed a functionally graded cylindrical shell model in the framework of the nonlocal strain gradient theory and the Gurtin-Murdoch surface elasticity theory. Three typical size effects including nonlocal effect, strain gradient effect, and surface energy effect on the free vibration were carried out. Vinh and Tounsi [35] analyzed the influence of the spatial variation of the nonlocal parameter on the free vibration of functionally graded sandwich nanoplates by modifying the classical nonlocal theory, and the motion equation was established based on the new inverse hyperbolic shear deformation theory and Hamilton's principle. The rigid body 
motion of the model itself is not taken into account in most literature on nanoplates. However, there are some phenomena similar to the motion at the macroscale [36] in nanosystems. For instance, medical nanorobots often need to move in the human blood as a whole to complete the auxiliary diagnosis and treatment of diseases through structural vibration. At this moment, kinematics and dynamics are coupled with each other. Of course, the out-ofplane vibration of a plate is more obvious than the in-plane vibration, and the nanoplate is no exception.

As mentioned above, functionally graded nanostructures tend to be designed for extreme working environments, and taking NEMS (e.g., nanosensors/resonators/nanorobots) as an example, they often need to work in some humid and hot environments. Therefore, it is of great significance to investigate the vibration characteristics of functionally graded nanoplates in the hygrothermal environment. In the investigation of mechanical behaviors of functionally gradient nanoplates, the hygrothermal effects have been attended by many researchers. Refrafi et al. [37] analyzed the buckling responses of simply supported functionally graded sandwich plates rested on the Winkler-Pasternak elastic foundation subjected to hygrothermal and mechanical loads. Tounsi et al. [38] proposed a simple four-variable trigonometric integral shear deformation model to perform the mechanical properties of advanced functionally graded ceramic-metal plates seated on a two-parameter elastic foundation, which exposed to a nonlinear hygro-thermomechanical load.

There are some performed studies on the axially moving nanoplates. In $[39,40]$, the nonlocal theory was adopted to analyze the free vibration of axially moving nanoplates, but the strain gradient effect was not taken into account. In [41, 42], the nonlocal strain gradient theory was employed to study the mechanical behaviors of axially moving nanoplates. However, sometimes, it is necessary to consider the hygrothermal effects because of the extreme working environments of functionally graded nanomaterials. For this purpose, the hygrothermal effect on the axially moving functionally graded nanoplates is considered herein. The novelty of this research is to explore the effects of the axial speed, gradient index, and internal and external characteristic parameters on the vibration of axially moving functionally graded nanoplates exposed to hygrothermal environments. In addition, the coupling effect between the internal characteristic parameters is also considered. The remaining parts of the present work are arranged as follows: In Section 2, the theoretical model of the axially moving functionally graded nanoplate subjected to hygrothermal loads is established, and the effect of the gradient index on the bending stiffness is demonstrated. Then, the partial differential governing equations describing the out-of-plane vibration are developed based on Hamilton's principle in the context of the nonlocal strain gradient theory. In Section 3, in order to calculate the natural frequencies, the composite beam function method in conjunction with the complex mode approach is employed according to the boundary conditions, and then the correctness of the approach is verified by comparisons. Afterwards, the effects of temperature variation, moisture concentration, axial speed, gradient index, nonlocal parameter, material characteristic scale parameter, and aspect ratio on the out-of-plane vibration frequency are analyzed via numerical examples. Finally, the summary is concluded in Section 4 . The results of the present work are of reference significance for the design, optimization, and control of axially moving MEMS/ NEMS based on functionally gradient nanoplates, such as nanorobots and nanosensors subjected to hygrothermal environments.

\section{Theoretical Constitutive and Governing Equations}

The core constitutive equations of the nonlocal strain gradient theory were expressed in an integral form initially [28]. However, the spatial integration of strains and its gradients is difficult to be solved. Hence, an equivalent differential constitutive equation was transformed for a two-dimensional nanostructure as

$$
\left[1-(e a)^{2} \nabla^{2}\right] \mathbf{t}=\mathbf{C}\left(1-l^{2} \nabla^{2}\right) \boldsymbol{\varepsilon}^{\prime},
$$

where $t$ is a total stress tensor, $\varepsilon$ I is a classical strain tensor, $\mathbf{C}$ is an elastic tensor, $\nabla^{2}$ is a Laplace operator, $a$ is a material internal scale, $l$ is a strain gradient characteristic scale, and $e$ is a nonlocal material parameter.

As depicted in Figure 1, given an axially moving functionally graded nanoplate with length $L_{a}$, width $L_{b}$, and thickness $h$, the $x-y$ plane coordinate system is established in the geometric middle plane and $z$ coordinate is along the thickness of the nanoplate. The axial speed is denoted by $v$. The functionally graded nanoplate is exposed to the hygrothermal environment with uniform temperature changing $\Delta T(K)$ and moisture concentration rise $\Delta H(\%)$, where $\Delta T=T-T_{0}$, in which $T$ is the applied temperature and $T_{0}=300 \mathrm{~K}$ is the reference temperature; $\Delta H=H-H_{0}$, in which $H$ is the applied moisture concentration and $H_{0}=0$ is the reference moisture. In the present work, we assume that the nanoplate is composed of the ceramic and metal, namely, the inner compositions of the two-dimensional thin nanostructure are the mixture of two materials by the gradient law so that the characteristics vary regularly according to the gradient index. Therefore, Young's modulus $E$, mass density $\rho$, thermal expansion coefficient $\alpha$, and moisture expansion coefficient $\beta$ of the functionally graded nanoplate can be respectively expressed as [43]

$$
\begin{aligned}
& E(z)=\left(E_{c}-E_{m}\right)\left(\frac{z}{h}+\frac{1}{2}\right)^{p}+E_{m}, \\
& \rho(z)=\left(\rho_{c}-\rho_{m}\right)\left(\frac{z}{h}+\frac{1}{2}\right)^{p}+\rho_{m}, \\
& \alpha(z)=\left(\alpha_{c}-\alpha_{m}\right)\left(\frac{z}{h}+\frac{1}{2}\right)^{p}+\alpha_{m}, \\
& \beta(z)=\left(\beta_{c}-\beta_{m}\right)\left(\frac{z}{h}+\frac{1}{2}\right)^{p}+\beta_{m} .
\end{aligned}
$$

where $p$ is the gradient index and two subscripts $c$ and $m$ denote the material properties of ceramic and metal phases, respectively. 


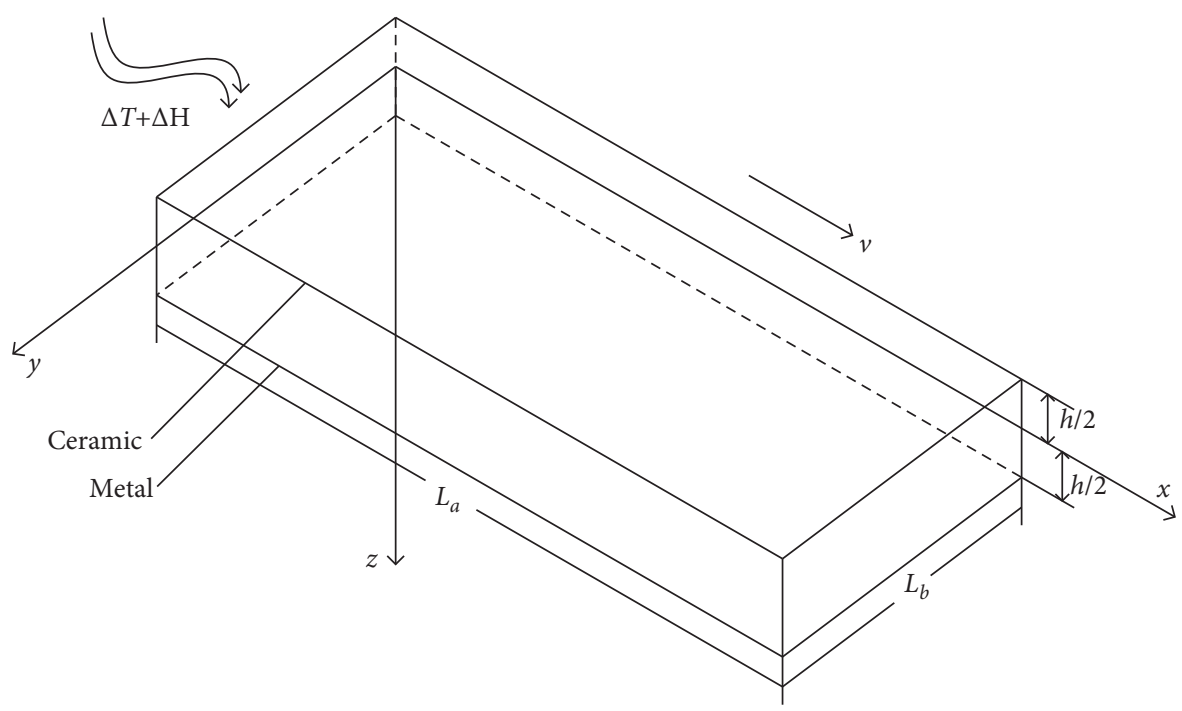

FIGURE 1: The schematic configuration of an axially moving functionally graded nanoplate in hygrothermal environments.

Because the changing trend of the above four types of material properties is identical, only taking Young's modulus of functionally graded nanoplates as an example, the variation along the thickness of the nanoplate is shown in Figure 2 under different gradient indexes, where $E_{c}=380 \mathrm{GPa}$ and $E_{m}=70 \mathrm{GPa}$ are adopted for ceramic and metal materials, respectively.

Let $u, v$, and $w$ represent the displacements of the nanoplate along $x, y$, and $z$ axes, respectively. Because the physical neutral layer is not equal to the geometric middle plane ( $x-y$ plane coordinate established herein) for an inhomogeneous functionally graded nanoplate, the position coordinate of the physical neutral layer is $z_{0}=\int_{-h / 2}^{h / 2} z E(z) \mathrm{d} z / \int_{-h / 2}^{h / 2} E(z) \mathrm{d} z$. Then, by applying the classic plate theory [44] and the concept of physical neutral plane [45], the displacement fields can be expressed as

$$
\begin{aligned}
& u(x, y, z, t)=-\left(z-z_{0}\right) \frac{\partial w(x, y, t)}{\partial x} \\
& v(x, y, z, t)=-\left(z-z_{0}\right) \frac{\partial w(x, y, t)}{\partial y} \\
& w(x, y, z, t)=w(x, y, t)
\end{aligned}
$$

According to the relationship between the classic Kirchhoff thin plate theory and the nonlocal strain gradient differential constitutive equation, the physical equations of the functionally graded nanoplate can be acquired as

$$
\left[1-(e a)^{2} \nabla^{2}\right]\left(\begin{array}{c}
\sigma_{x x} \\
\sigma_{y y} \\
\tau_{x y}
\end{array}\right)=\left(1-l^{2} \nabla^{2}\right) B\left[\begin{array}{ccc}
1 & \mu & 0 \\
\mu & 1 & 0 \\
0 & 0 & (1-\mu) / 2
\end{array}\right]\left(\begin{array}{c}
\varepsilon_{x x} \\
\varepsilon_{y y} \\
\gamma_{x y}
\end{array}\right),
$$

where the functionally graded nanoplate is regarded as a plane stress problem, $B=E(z) /\left(1-\mu^{2}\right)$, and $\mu$ is Poisson's ratio.
It is noted that the definition of the bending moment and its relationship with stress are valid in both macroscale and microscale. Therefore, the defining expression of the bending moment in the nonlocal strain gradient theory is consistent with that in the classical continuum theory. The difference is that the stress in the definition is nonlocal stress and classical stress, respectively. Then, by applying the classic plate theory [44], the bending moment can be expressed as $\left(M_{x x}, M_{x y}, M_{y y}\right)=\int_{-h / 2}^{h / 2}\left(\sigma_{x x}, \sigma_{x y}, \sigma_{y y}\right)\left(z-z_{0}\right) \mathrm{d} z$

According to equation (3), the relationship between the bending moment and the displacement can be derived from equation (4) as

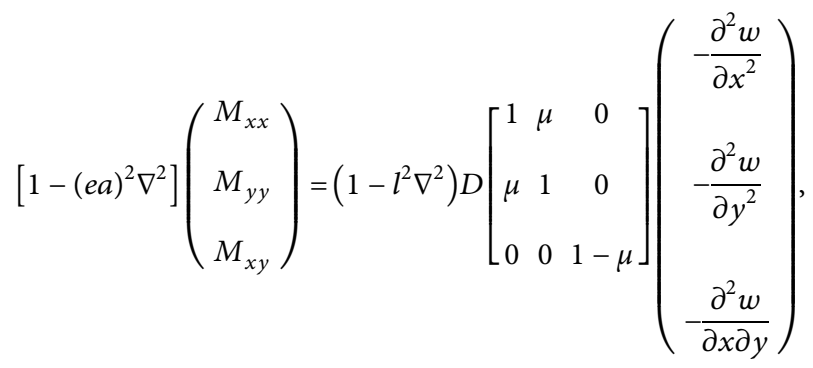

where $w$ represents the out-of-plane displacement and $D=$ $\int_{-h / 2}^{h / 2} B\left(z-z_{0}\right)^{2} \mathrm{~d} z$ is the equivalent bending stiffness. The effect of the gradient index on the equivalent bending stiffness is shown in Figure 3.

As clearly visible in Figure 3, when the gradient index increases, the equivalent bending stiffness decreases quickly at first and then gradually slows down, which indicates that a higher gradient index reduces the equivalent bending stiffness of functionally graded nanoplates. The observations can provide reference for the design of functionally graded nanoplates subjected to different working conditions.

To analyze the out-of-plane vibration of axially moving functionally graded nanoplates in hygrothermal 
environments, Hamilton's principle is utilized to derive the governing equation of motion, which requires

$$
\int_{t_{1}}^{t_{2}}(\delta U+\delta V-\delta K) \mathrm{d} t=0
$$

where $\delta U, \delta V$, and $\delta K$ are the variations of the strain potential energy, external potential energy, and kinetic energy, respectively, and $t_{1}$ and $t_{2}$ represent two arbitrary moments. Firstly, the strain potential energy for the nanoplate may be calculated as

$$
\begin{aligned}
\int_{t_{1}}^{t_{2}} \delta U d t= & -\int_{t_{1}}^{t_{2}} \iint_{A}\left(M_{x x} \delta \frac{\partial^{2} w}{\partial x^{2}}+M_{y y} \delta \frac{\partial^{2} w}{\partial y^{2}}+2 M_{x y} \delta \frac{\partial^{2} w}{\partial x \partial y}\right) \mathrm{d} A \mathrm{~d} t \\
= & -\left.\int_{t_{1}}^{t_{2}} \int M_{x x} \delta \frac{\partial w}{\partial x}\right|_{0} ^{L_{a}} \mathrm{~d} y \mathrm{~d} t+\left.\int_{t_{1}}^{t_{2}} \int \frac{\partial M_{x x}}{\partial x} \delta w\right|_{0} ^{L_{a}} \mathrm{~d} y \mathrm{~d} t-\int_{t_{1}}^{t_{2}} \iint_{A} \frac{\partial^{2} M_{x x}}{\partial x^{2}} \delta w \mathrm{~d} A \mathrm{~d} t \\
& -\left.\int_{t_{1}}^{t_{2}} \int M_{y y} \delta \frac{\partial w}{\partial y}\right|_{0} ^{L_{b}} \mathrm{~d} x \mathrm{~d} t+\left.\int_{t_{1}}^{t_{2}} \int \frac{\partial M_{y y}}{\partial y} \delta w\right|_{0} ^{L_{b}} \mathrm{~d} x \mathrm{~d} t-\int_{t_{1}}^{t_{2}} \iint_{A} \frac{\partial^{2} M_{y y}}{\partial y^{2}} \delta w \mathrm{~d} A \mathrm{~d} t \\
& -\left.\int_{t_{1}}^{t_{2}} \int M_{x y} \delta \frac{\partial w}{\partial y}\right|_{0} ^{L_{a}} \mathrm{~d} y \mathrm{~d} t+\left.\int_{t_{1}}^{t_{2}} \int \frac{\partial M_{x y}}{\partial x} \delta w\right|_{0} ^{L_{b}} \mathrm{~d} x \mathrm{~d} t-2 \int_{t_{1}}^{t_{2}} \iint_{A} \frac{\partial^{2} M_{x y}}{\partial x \partial y} \delta w \mathrm{~d} A \mathrm{~d} t-\left.\int_{t_{1}}^{t_{2}} \int M_{x y} \delta \frac{\partial w}{\partial x}\right|_{0} ^{L_{b}} \mathrm{~d} y \mathrm{~d} t+\left.\int_{t_{1}}^{t_{2}} \int \frac{\partial M_{x y}}{\partial y} \delta w\right|_{0} ^{L_{a}} \mathrm{~d} x \mathrm{~d} t,
\end{aligned}
$$

where $A$ is the region of the middle plane of the nanoplate. Then, we assume that the temperature changing results in the external potential energy which is reverse to work done by external loads, and it can be written as

$$
\begin{aligned}
\int_{t_{1}}^{t_{2}} \delta V d t= & -\int_{t_{1}}^{t_{2}} \iint_{A}\left(N_{x} \frac{\partial w}{\partial x} \delta \frac{\partial w}{\partial x}+N_{y} \frac{\partial w}{\partial y} \delta \frac{\partial w}{\partial y}\right) \mathrm{d} A \mathrm{~d} t \\
= & -\left.\int_{t_{1}}^{t_{2}} \int N_{x} \frac{\partial w}{\partial x} \delta w\right|_{0} ^{L_{a}} \mathrm{~d} y \mathrm{~d} t+\int_{t_{1}}^{t_{2}} \iint_{A} N_{x} \frac{\partial^{2} w}{\partial x^{2}} \delta w \mathrm{~d} A \mathrm{~d} t \\
& -\left.\int_{t_{1}}^{t_{2}} \int N_{y} \frac{\partial w}{\partial y} \delta w\right|_{0} ^{L_{b}} \mathrm{~d} x \mathrm{~d} t+\int_{t_{1}}^{t_{2}} \iint_{A} N_{y} \frac{\partial^{2} w}{\partial x^{2}} \delta w \mathrm{~d} A \mathrm{~d} t
\end{aligned}
$$

where $N_{x}=N_{y}=N_{T}+N_{H}$ are the hygrothermal loads, in which $N_{T}$ is the temperature load and $N_{H}$ is the humid environment load, respectively. And, the hygrothermal resultant can be expressed by

$$
\begin{aligned}
N_{T} & =\int_{-(h / 2)}^{h / 2} E(z) \alpha(z) \Delta T \mathrm{~d} z=n_{T} \Delta T h, \\
N_{H} & =\int_{-(h / 2)}^{h / 2} E(z) \beta(z) \Delta H \mathrm{~d} z=n_{H} \Delta H h,
\end{aligned}
$$

in which

$$
\begin{aligned}
& n_{T}=\frac{\left[E_{c}+\left(E_{c}+E_{m}\right) p\right] \alpha_{c}+p\left(E_{c}+2 E_{m} p\right) \alpha_{m}}{(1+p)(1+2 p)}, \\
& n_{H}=\frac{\left[E_{c}+\left(E_{c}+E_{m}\right) p\right] \beta_{c}+p\left(E_{c}+2 E_{m} p\right) \beta_{m}}{(1+p)(1+2 p)} .
\end{aligned}
$$

Finally, the kinetic energy of the nanoplate is given by

$$
\begin{aligned}
\int_{t_{1}}^{t_{2}} \delta K \mathrm{~d} t= & \int_{t_{1}}^{t_{2}} \iint_{A} I_{0}\left(\frac{\partial w}{\partial t}+v \frac{\partial w}{\partial x}\right) \delta\left(\frac{\partial w}{\partial t}+v \frac{\partial w}{\partial x}\right) \mathrm{d} A \mathrm{~d} t \\
= & \left.\iint_{A} I_{0} h \frac{\partial w}{\partial t} \delta w\right|_{t_{1}} ^{t_{2}} \mathrm{~d} A-\int_{t_{1}}^{t_{2}} \iint_{A} I_{0} \frac{\partial^{2} w}{\partial t^{2}} \delta w \mathrm{~d} A \mathrm{~d} t \\
& +\left.\int_{t_{1}}^{t_{2}} \int I_{0} \frac{\partial w}{\partial t} v \delta w\right|_{0} ^{L_{a}} \mathrm{~d} y \mathrm{~d} t-\int_{t_{1}}^{t_{2}} \iint_{A} I_{0} v \frac{\partial^{2} w}{\partial x \partial t} \delta w \mathrm{~d} A \mathrm{~d} t \\
& +\left.\iint_{A} I_{0} v \frac{\partial w}{\partial x} \delta w\right|_{t_{1}} ^{\mathrm{d} A} A-\int_{t_{1}}^{t_{2}} \iint_{A}^{t_{2}} I_{0} v \frac{\partial^{2} w}{\partial x \partial t} \delta w \mathrm{~d} A \mathrm{~d} t+\left.\int_{t_{1}}^{t_{2}} \int I_{0} v^{2} \frac{\partial w}{\partial x} \delta w\right|_{0} ^{L_{a}} \mathrm{~d} y \mathrm{~d} t-\int_{t_{1}}^{t_{2}} \iint_{A} I_{0} v^{2} \frac{\partial^{2} w}{\partial x^{2}} \delta w \mathrm{~d} A \mathrm{~d} t
\end{aligned}
$$




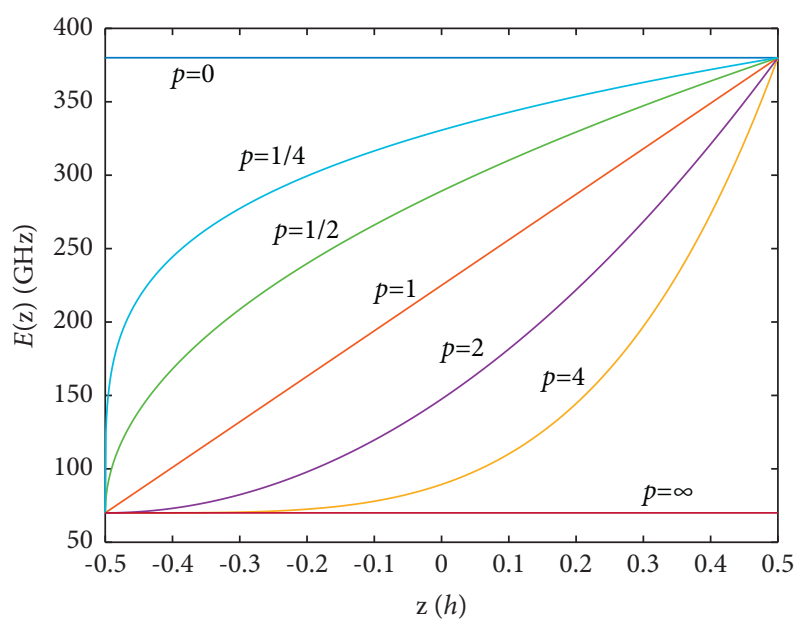

Figure 2: Variation of material Young's modulus along thickness.

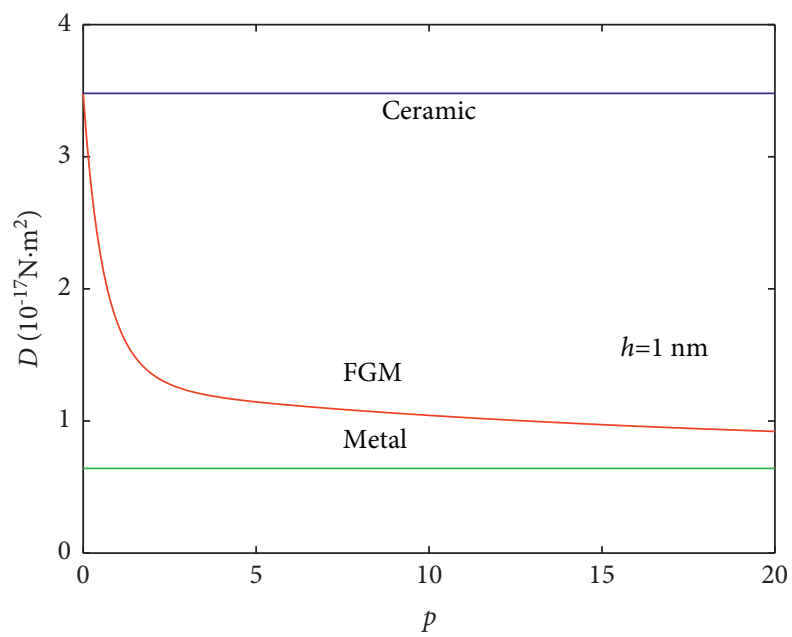

FIGURE 3: Effects of the gradient index on the equivalent bending stiffness.

where $I_{0}=\int_{-h / 2}^{h / 2} \rho(z) d z[45]$.

Substituting equations (7), (8), and (11) into equation (6), and then integrating by parts, the partial differential equation that governs the out-of-plane vibration can be written as

$$
\begin{array}{r}
\frac{\partial^{2} M_{x x}}{\partial x^{2}}+\frac{\partial^{2} M_{y y}}{\partial y^{2}}+2 \frac{\partial^{2} M_{x y}}{\partial x \partial y}-\left(N_{T}+N_{H}\right)\left(\frac{\partial^{2} w}{\partial x^{2}}+\frac{\partial^{2} w}{\partial y^{2}}\right) \\
=I_{0}\left(v^{2} \frac{\partial^{2} w}{\partial x^{2}}+2 v \frac{\partial^{2} w}{\partial x \partial t}+\frac{\partial^{2} w}{\partial t^{2}}\right) .
\end{array}
$$

According to the variational principle, the essential/ natural boundary conditions of the model along $x=0, L_{a}$ can be obtained as

$$
w=0 \text { or } \frac{\partial M_{x x}}{\partial x}+\frac{\partial M_{x y}}{\partial y}-N_{x} \frac{\partial w}{\partial x}-I_{0} \frac{\partial w}{\partial t} v-I_{0} v^{2} \frac{\partial w}{\partial x}=0,
$$

$\frac{\partial w}{\partial x}=0$ or $M_{x x}=0$,

$\frac{\partial w}{\partial y}=0$ or $M_{x y}=0$. 
And the boundary conditions along $y=0, L_{b}$ are acquired as

$$
\begin{aligned}
w & =0 \text { or } \frac{\partial M_{y y}}{\partial y}+\frac{\partial M_{x y}}{\partial x}-N_{y} \frac{\partial w}{\partial y}=0 \\
\frac{\partial w}{\partial x} & =0 \text { or } M_{x y}=0 \\
\frac{\partial w}{\partial y} & =0 \text { or } M_{y y}=0
\end{aligned}
$$

Substituting equation (5) into (12) and the governing equation in terms of out-of-plane displacement can be expressed as

$$
\begin{aligned}
& \left(1-l^{2} \nabla^{2}\right) D\left(\frac{\partial^{4} w}{\partial x^{4}}+\frac{\partial^{4} w}{\partial y^{4}}+2 \frac{\partial^{4} w}{\partial x^{2} \partial y^{2}}\right) \\
& +\left[1-(e a)^{2} \nabla^{2}\right]\left[\left(N_{T}+N_{H}\right)\left(\frac{\partial^{2} w}{\partial x^{2}}+\frac{\partial^{2} w}{\partial y^{2}}\right)+I_{0}\left(v^{2} \frac{\partial^{2} w}{\partial x^{2}}+2 v \frac{\partial^{2} w}{\partial x \partial t}+\frac{\partial^{2} w}{\partial t^{2}}\right)\right]=0 .
\end{aligned}
$$

In order to obtain the dimensionless governing equation and analyze the out-of-plane vibration characteristics of axially moving functionally graded nanoplates qualitatively, the following variables and parameters are necessary:

$$
\begin{aligned}
& \bar{x}=\frac{x}{L_{a}}, \bar{y}=\frac{y}{L_{b}}, \bar{w}=\frac{w}{h}, \lambda=\frac{L_{a}}{L_{b}}, \bar{N}_{T}=\frac{N_{T} L_{a}^{2}}{D}, \bar{N}_{H}=\frac{N_{H} L_{a}^{2}}{D}, \\
& \tau=\frac{e a}{L_{a}}, \zeta=\frac{l}{L_{a}}, \xi=\sqrt{\frac{I_{0}}{D}} L_{a} v, \bar{t}=\sqrt{\frac{D}{I_{0} L_{a}^{4}} t .}
\end{aligned}
$$

$$
\begin{aligned}
& \zeta^{2} \frac{\partial^{6} \bar{w}}{\partial \bar{x}^{6}}+3 \zeta^{2} \lambda^{2} \frac{\partial^{6} \bar{w}}{\partial \bar{x}^{4} \partial \bar{y}^{2}}+3 \zeta^{2} \lambda^{4} \frac{\partial^{6} \bar{w}}{\partial \bar{x}^{2} \partial \bar{y}^{4}}+\zeta^{2} \lambda^{6} \frac{\partial^{6} \bar{w}}{\partial \bar{y}^{6}} \\
& +\left[\tau^{2}\left(\bar{N}_{T}+\bar{N}_{H}+\xi^{2}\right)-1\right] \frac{\partial^{4} \bar{w}}{\partial \bar{x}^{4}}+\left[\tau^{2} \lambda^{2}\left(2 \bar{N}_{T}+2 \bar{N}_{H}+\xi^{2}\right)-2 \lambda^{2}\right] \frac{\partial^{4} \bar{w}}{\partial \bar{x}^{2} \partial \bar{y}^{2}} \\
& +\lambda^{4}\left[\tau^{2}\left(\bar{N}_{T}+\bar{N}_{H}\right)-1\right] \frac{\partial^{4} \bar{w}}{\partial \bar{y}^{4}}+2 \tau^{2} \xi \frac{\partial^{4} \bar{w}}{\partial \bar{x}^{3} \partial \bar{t}}+\tau^{2} \frac{\partial^{4} \bar{w}}{\partial \bar{x}^{2} \partial \bar{t}^{2}} \\
& +2 \tau^{2} \xi \lambda^{2} \frac{\partial^{4} \bar{w}}{\partial \bar{x} \partial \bar{y}^{2} \partial \bar{t}}+\tau^{2} \lambda^{2} \frac{\partial^{4} \bar{w}}{\partial \bar{y}^{2} \partial \bar{t}^{2}}-\left(\bar{N}_{T}+\bar{N}_{H}+\xi^{2}\right) \frac{\partial^{2} \bar{w}}{\partial \bar{x}^{2}} \\
& -\left(\bar{N}_{T}+\bar{N}_{H}\right) \lambda^{2} \frac{\partial^{2} \bar{w}}{\partial \bar{y}^{2}}-2 \xi \frac{\partial^{2} \bar{w}}{\partial \bar{x} \partial \bar{t}}-\frac{\partial^{2} \bar{w}}{\partial \bar{t}^{2}}=0 .
\end{aligned}
$$


The spatial variables $\bar{x}$ and $\bar{y}$ and time variable $\bar{t}$ are coupled in equation (17). In the linear elasticity and small deformation, one can separate these variables. The solution of out-of-plane displacement is set to

$$
\bar{w}(\bar{x}, \bar{y}, \bar{t})=\bar{W}(\bar{x}, \bar{y}) e^{i \omega_{m n} \bar{t}}
$$

where $\omega_{m n}$ is the vibration circular frequency, usually a complex frequency, of which the real and imaginary components can be used to describe the vibration frequency and related stability, respectively.

Substituting equation (18) into (17), the nondimensional frequency-domain governing equation can be obtained as

$$
\begin{aligned}
& \zeta^{2} \frac{\partial^{6} \bar{W}}{\partial \bar{x}^{6}}+3 \zeta^{2} \lambda^{2} \frac{\partial^{6} \bar{W}}{\partial \bar{x}^{4} \partial \bar{y}^{2}}+3 \zeta^{2} \lambda^{4} \frac{\partial^{6} \bar{W}}{\partial \bar{x}^{2} \partial \bar{y}^{4}}+\zeta^{2} \lambda^{6} \frac{\partial^{6} \bar{W}}{\partial \bar{y}^{6}} \\
& +\left[\tau^{2}\left(\bar{N}_{T}+\bar{N}_{H}+\xi^{2}\right)-1\right] \frac{\partial^{4} \bar{W}}{\partial \bar{x}^{4}}+\left[\tau^{2} \lambda^{2}\left(2 \bar{N}_{T}+2 \bar{N}_{H}+\xi^{2}\right)-2 \lambda^{2}\right] \frac{\partial^{4} \bar{W}}{\partial \bar{x}^{2} \partial \bar{y}^{2}} \\
& +\lambda^{4}\left[\tau^{2}\left(\bar{N}_{T}+\bar{N}_{H}\right)-1\right] \frac{\partial^{4} \bar{W}}{\partial \bar{y}^{4}}+2 i \tau^{2} \xi \omega_{m n} \frac{\partial^{3} \bar{W}}{\partial \bar{x}^{3}}+2 i \tau^{2} \xi \lambda^{2} \omega_{m n} \frac{\partial^{3} \bar{W}}{\partial \bar{x} \partial \bar{y}^{2}} \\
& -\left(\bar{N}_{T}+\bar{N}_{H}+\xi^{2}+\tau^{2} \omega_{m n}^{2}\right) \frac{\partial^{2} \bar{W}}{\partial \bar{x}^{2}}-\lambda^{2}\left(\bar{N}_{T}+\bar{N}_{H}+\tau^{2} \omega_{m n}^{2}\right) \frac{\partial^{2} \bar{W}}{\partial \bar{y}^{2}} \\
& -2 i \xi \omega_{m n} \frac{\partial \bar{W}}{\partial \bar{x}}+\omega_{m n}^{2} \bar{W}=0 .
\end{aligned}
$$

\section{Numerical Results and Discussion}

Unlike most research studies applying Galerkin method to investigate the moving nanosystems $[39,41,46]$, the present work employs the composite beam function method [47] to develop the dynamic stability of axially moving nanoplates. The approach assumes the displacement function according to boundary conditions, so that the governing equation can be solved numerically directly. Compared with other accurate solution methods such as Galerkin and Navier's solution, the advantage of the present method is that it can analyze systems with various boundary conditions.
In addition to the governing equations of axially moving functionally graded nanoplates established in the previous section, the boundary conditions are needed to solve the outof-plane vibration theoretical model. Taking the edge constraint of simply supported on four sides as an example, according to equations (13) and (14), the corresponding natural boundary conditions at $x=0, L_{a}$ are chosen as $M_{x x}=0$, and the natural boundary conditions at $y=0, L_{b}$ are chosen as $M_{y y}=0$. Then, considering the relationship between bending moment and displacement in equation (5), the details of the basic boundary conditions of the nanoplates can be obtained as

$$
\begin{aligned}
& \text { Essential }: w=\frac{\partial w}{\partial y}=\frac{\partial^{2} w}{\partial x^{2}}=\left.\frac{\partial^{2} w}{\partial y^{2}}\right|_{x=0, L_{a}}=0, \quad w=\frac{\partial w}{\partial x}=\frac{\partial^{2} w}{\partial x^{2}}=\left.\frac{\partial^{2} w}{\partial y^{2}}\right|_{y=0, L_{b}}=0 \\
& \text { Natural : }-\left.D\left(\frac{\partial^{2} w}{\partial x^{2}}-l^{2} \frac{\partial^{4} w}{\partial x^{4}}\right)\right|_{x=0, L_{a}}=0, \quad-\left.D\left(\frac{\partial^{2} w}{\partial y^{2}}-l^{2} \frac{\partial^{4} w}{\partial y^{4}}\right)\right|_{y=0, L_{b}}=0
\end{aligned}
$$


Therefore, the dimensionless boundary conditions can be written as

$$
\begin{aligned}
& \bar{W}(\bar{x}, \bar{y})=\frac{\partial \bar{W}(\bar{x}, \bar{y})}{\partial \bar{y}}=\frac{\partial^{2} \bar{W}(\bar{x}, \bar{y})}{\partial \bar{x}^{2}}=\frac{\partial^{2} \bar{W}(\bar{x}, \bar{y})}{\partial \bar{y}^{2}}=\left.\frac{\partial^{4} \bar{W}(\bar{x}, \bar{y})}{\partial \bar{x}^{4}}\right|_{\bar{x}=0,1}=0 \\
& \bar{W}(\bar{x}, \bar{y})=\frac{\partial \bar{W}(\bar{x}, \bar{y})}{\partial \bar{x}}=\frac{\partial^{2} \bar{W}(\bar{x}, \bar{y})}{\partial \bar{x}^{2}}=\frac{\partial^{2} \bar{W}(\bar{x}, \bar{y})}{\partial \bar{y}^{2}}=\left.\frac{\partial^{4} \bar{W}(\bar{x}, \bar{y})}{\partial \bar{y}^{4}}\right|_{\bar{y}=0,1}=0 .
\end{aligned}
$$

Utilizing the composite beam function method [47], the dimensionless out-of-plane displacement can be assumed as

$$
\bar{W}(\bar{x}, \bar{y})=\sum_{m=1}^{M} \sum_{n=1}^{N} A_{m n} \psi_{m n}(\bar{x}, \bar{y})=\sum_{m=1}^{M} \sum_{n=1}^{N} A_{m n} \phi_{m}(\bar{x}) \varphi_{n}(\bar{y}),
$$

where $\phi_{m}(\bar{x})$ and $\varphi_{n}(\bar{y})$ are the adopted mode functions of $x$ and $y$ directions, respectively, and correspondingly, $\psi_{m n}(\bar{x}, \bar{y})$ is the product of two mode functions.
For the nanoplate simply supported on four sides, the $y$ direction mode function can be defined as a sine function that satisfies the boundary conditions in equation (21). Hence, the product of mode functions can be expressed as

$$
\psi_{m n}(\bar{x}, \bar{y})=\phi_{m}(\bar{x}) \sin n \pi \bar{y} .
$$

Substituting equation (23) into (22), and further into equation (19) yields

$$
\begin{aligned}
& \zeta^{2} \frac{\partial^{6} \phi_{m}}{\partial \bar{x}^{6}}+\left[\tau^{2}\left(\bar{N}_{T}+\bar{N}_{H}+\xi^{2}\right)-1-3(n \pi \lambda)^{2} \zeta^{2}\right] \frac{\partial^{4} \phi_{m}}{\partial \bar{x}^{4}}+2 i \tau^{2} \xi \omega_{m n} \frac{\partial^{3} \phi_{m}}{\partial \bar{x}^{3}} \\
& +\left\{(n \pi \lambda)^{2}\left[2-\tau^{2}\left(2 \bar{N}_{T}+2 \bar{N}_{H}+\xi^{2}\right)\right]+3(n \pi \lambda)^{4} \zeta^{2}-\left(\bar{N}_{T}+\bar{N}_{H}+\xi^{2}+\tau^{2} \omega_{m n}^{2}\right)\right\} \frac{\partial^{2} \phi_{m}}{\partial \bar{x}^{2}} \\
& -2 i \xi \omega_{m n}\left[(n \pi \lambda)^{2} \tau^{2}+1\right] \frac{\partial \phi_{m}}{\partial \bar{x}} \\
& +\left[(n \pi \lambda)^{4}\left(\tau^{2} \bar{N}_{T}+\tau^{2} \bar{N}_{H}-1\right)+(n \pi \lambda)^{2}\left(\bar{N}_{T}+\bar{N}_{H}+\tau^{2} \omega_{m n}^{2}\right)+\omega_{m n}^{2}-(n \pi \lambda)^{6} \zeta^{2}\right] \phi_{m}=0 .
\end{aligned}
$$

The solution to equation (24) can be written in form as $\phi_{m}(\bar{x})=C e^{i \beta \bar{x}}$, and the following equation can be obtained by substituting the solution into equation (24):

$$
\begin{aligned}
& \zeta^{2} \beta^{6}+\left[3(n \pi \lambda)^{2} \zeta^{2}-\tau^{2}\left(\bar{N}_{T}+\bar{N}_{H}+\xi^{2}\right)+1\right] \beta^{4}-2 \tau^{2} \xi \omega_{m n} \beta^{3} \\
& +\left\{(n \pi \lambda)^{2}\left[2-\tau^{2}\left(2 \bar{N}_{T}+2 \bar{N}_{H}+\xi^{2}\right)\right]+3(n \pi \lambda)^{4} \zeta^{2}-\left(\bar{N}_{T}+\bar{N}_{H}+\xi^{2}+\tau^{2} \omega_{m n}^{2}\right)\right\} \beta^{2} \\
& -2 \xi \omega_{m n}\left[(n \pi \lambda)^{2} \tau^{2}+1\right] \beta \\
& +\left[(n \pi \lambda)^{6} \zeta^{2}-(n \pi \lambda)^{4}\left(\tau^{2} \bar{N}_{T}+\tau^{2} \bar{N}_{H}-1\right)-(n \pi \lambda)^{2}\left(\bar{N}_{T}+\bar{N}_{H}+\tau^{2} \omega_{m n}^{2}\right)-\omega_{m n}^{2}\right]=0
\end{aligned}
$$

As a sixth-order ordinary differential equation, the solution to equation in (25) can be assumed as

$$
\phi_{m}(\bar{x})=C_{1 m}\left(e^{i \beta_{1 m} \bar{x}}+C_{2 m} e^{i \beta_{2 m} \bar{x}}+C_{3 m} e^{i \beta_{3 m} \bar{x}}+C_{4 m} e^{i \beta_{4 m} \bar{x}}+C_{5 m} e^{i \beta_{5 m} \bar{x}}+C_{6 m} e^{i \beta_{6 m} \bar{x}}\right),
$$


where $C_{j m}(j=1,2,3, \ldots ., 6)$ denotes six unknown coefficients introduced by integral, and $\beta_{j m}(j=1,2,3, \ldots \ldots, 6)$ are corresponding six roots of algebraic equation (25). According to equation (21), the boundary conditions can be written as

$$
\phi_{m}(0)=\phi_{m}(1)=\frac{\partial^{2} \phi_{m}(0)}{\partial \bar{x}^{2}}=\frac{\partial^{2} \phi_{m}(1)}{\partial \bar{x}^{2}}=\frac{\partial^{4} \phi_{m}(0)}{\partial \bar{x}^{4}}=\frac{\partial^{4} \phi_{m}(1)}{\partial \bar{x}^{4}}=0 .
$$

As a result, a set of algebraic equations are acquired by substituting equation (26) into the simplified boundary condition equation (27). To ensure the existence of the nontrivial solutions of the coefficients or mode function in equation (26), the determinant of the coefficient matrix in the algebraic equations should be set to zero.

$$
\left|\begin{array}{cccccc}
1 & 1 & 1 & 1 & 1 & 1 \\
e^{i \beta_{1 m}} & e^{i \beta_{2 m}} & e^{i \beta_{3 m}} & e^{i \beta_{4 m}} & e^{i \beta_{5 m}} & e^{i \beta_{6 m}} \\
i \beta^{2} & i \beta^{2}{ }_{2 m} & i \beta_{3 m}^{2} & i \beta^{2} & i \beta^{2}{ }_{5 m} & i \beta_{6 m}^{2} \\
i \beta^{2}{ }_{1 m} e^{i \beta_{1 m}} & i \beta_{2 m}^{2} e^{i \beta_{2 m}} & i \beta_{3 m}^{2} e^{i \beta_{3 m}} & i \beta^{2}{ }_{4 m} e^{i \beta_{4 m}} & i \beta^{2}{ }_{5 m} e^{i \beta_{5 m}} & i \beta_{6 m}^{2} e^{i \beta_{6 m}} \\
\beta_{1 m}^{4} & \beta_{2 m}^{4} & \beta_{3 m}^{4} & \beta_{4 m}^{4} & \beta_{5 m}^{4} & \beta_{6 m}^{4} \\
\beta_{1 m}^{4} e^{i \beta_{1 m}} & \beta_{2 m}^{4} e^{i \beta_{2 m}} & \beta_{3 m}^{4} e^{i \beta_{3 m}} & \beta_{4 m}^{4} e^{i \beta_{4 m}} & \beta_{5 m}^{4} e^{i \beta_{5 m}} & \beta_{6 m}^{4} e^{i \beta_{6 m}}
\end{array}\right|=0
$$

Combining equations (25) and (28), the dimensionless vibration frequency can be determined numerically. In the calculation process, the other parameters are set as constant values firstly; then, an interval of the remaining parameter is provided, so that the variation of vibration frequencies with respect to the remaining parameter can be achieved via a numerical calculation program. Consequently, the relationships between the dimensionless vibration frequency and each parameter can be obtained.

In order to demonstrate the accuracy and validity of the present analyses, a benchmark result is given by the comparison with previous results available in the literature. First, by ignoring the strain gradient, axial velocity, and hygrothermal environment effects, the present results are compared with those reported by Natarajan et al. [48] and Jandaghian and Rahmani [49] in Table 1. The results agree well with those from the literature, which proves that the model and method proposed in this paper are reliable in vibration analyses. In addition, the influence of uniform thermal effects on nanoplates is also compared in Table 2 [50]. The verification example reveals that the proposed approach can accurately predict the behavior of nanoplates under the influence of external environmental loads.

In the present examples, we take $n=1$, and the material properties of the functionally graded nanoplate are shown as follows [51].

$$
\begin{aligned}
E_{c} & =380 \mathrm{GPa}, \\
\mu_{c} & =0.3, \\
\rho_{c} & =3800 \mathrm{~kg} / \mathrm{m}^{3}, \\
\alpha_{c} & =7 \times 10^{-6} \mathrm{~K}^{-1}, \\
\beta_{c} & =0.001 \%, \\
E_{m} & =70 \mathrm{GPa}, \\
\mu_{m} & =0.3, \\
\rho_{m} & =2702 \mathrm{~kg} / \mathrm{m}^{3}, \\
\alpha_{m} & =23 \times 10^{-6} \mathrm{~K}^{-1}, \\
\beta_{m} & =0.44 \% .
\end{aligned}
$$

As for the magnitude of the nonlocal parameter $e a$ and the strain gradient parameter $l$, some literature $[52,53]$ has made a special discussion, but there are no experimental methods to determine it. In the physical sense, these two parameters represent the continuous range of nonlocal longrange action and the strength of strain gradient influence. In the present work, following the method used by Lu et al. [54], the two length parameters are normalized by length $L_{a}$ to obtain numerical results, and it is used that both $e a / L_{a}$ and $l / L_{a}$ are in the range of 0 to 0.2 .

Firstly, the influences of the hygrothermal environment on vibrating functionally graded nanoplates are examined. 
TABLE 1: Comparison of nondimensional fundamental natural frequencies of FG nanoplates.

\begin{tabular}{|c|c|c|c|c|c|c|c|c|}
\hline \multirow{2}{*}{$L_{\mathrm{a}} / L_{\mathrm{b}}$} & \multirow{2}{*}{$L_{\mathrm{a}} / h$} & \multirow{2}{*}{$(e a)^{2}$} & \multicolumn{3}{|c|}{ Mode 1} & \multicolumn{3}{|c|}{ Mode 2} \\
\hline & & & Ref. [48] & Ref. [49] & Present & Ref. [48] & Ref. [49] & Present \\
\hline \multirow{8}{*}{1} & \multirow{4}{*}{10} & 0 & 0.0441 & 0.0458 & 0.0458 & 0.1051 & 0.1127 & 0.1144 \\
\hline & & 1 & 0.0403 & 0.04200420 & 0.0418 & 0.0860 & 0.0934 & 0.0936 \\
\hline & & 2 & 0.0374 & 0.0390 & 0.0388 & 0.0745 & 0.0814 & 0.0812 \\
\hline & & 4 & 0.0330 & 0.0345 & 0.0342 & 0.0609 & 0.0670 & 0.0664 \\
\hline & \multirow{4}{*}{20} & 0 & 0.0113 & 0.0115 & 0.0114 & 0.0278 & 0.0286 & 0.0286 \\
\hline & & 1 & 0.0103 & 0.0976 & 0.0105 & 0.0228 & 0.0235 & 0.0234 \\
\hline & & 2 & 0.0096 & 0.0098 & 0.0097 & 0.0197 & 0.0204 & 0.0203 \\
\hline & & 4 & 0.0085 & 0.0086 & 0.0086 & 0.0161 & 0.0167 & 0.0166 \\
\hline \multirow{8}{*}{2} & \multirow{4}{*}{10} & 0 & 0.1055 & 0.1127 & 0.1144 & 0.1615 & 0.1795 & 0.1831 \\
\hline & & 1 & 0.0863 & 0.0934 & 0.0936 & 0.1208 & 0.1376 & 0.1396 \\
\hline & & 2 & 0.0748 & 0.081400 .0 & 0.0812 & 0.1006 & 0.1158 & 0.1140 \\
\hline & & 4 & 0.0612 & 0.0670 & 0.0664 & 0.0793 & 0.0920 & 0.0898 \\
\hline & \multirow{4}{*}{20} & 0 & 0.0279 & 0.0286 & 0.0286 & 0.0440 & 0.0458 & 0.0458 \\
\hline & & 1 & 0.0229 & 0.0235 & 0.0234 & 0.0329 & 0.0345 & 0.0342 \\
\hline & & 2 & 0.0198 & 0.0204 & 0.0203 & 0.0274 & 0.0288 & 0.0285 \\
\hline & & 4 & 0.0162 & 0.0167 & 0.0166 & 0.0216 & 0.0227 & 0.0224 \\
\hline
\end{tabular}

TABLE 2: Comparison of nondimensional fundamental natural frequencies of FG nanoplates subjected to uniform temperature rise.

\begin{tabular}{|c|c|c|c|c|c|c|c|}
\hline \multirow{2}{*}{$L_{\mathrm{a}} / L_{\mathrm{b}}$} & \multirow{2}{*}{$(e a)^{2}$} & \multicolumn{2}{|c|}{$\Delta T=20 \mathrm{~K}$} & \multicolumn{2}{|c|}{$\Delta T=50 \mathrm{~K}$} & \multicolumn{2}{|c|}{$\Delta T=100 \mathrm{~K}$} \\
\hline & & Ref. [50] & Present & Ref. [50] & Present & Ref. [50] & Present \\
\hline \multirow{3}{*}{1} & 0 & 3.40990 & 3.44383 & 3.20129 & 3.33459 & 2.79383 & 3.14409 \\
\hline & 1 & 3.09232 & 3.13424 & 2.86011 & 3.0138 & 2.39481 & 2.80157 \\
\hline & 2 & 2.84287 & 2.89195 & 2.58795 & 2.76059 & 2.06147 & 2.52759 \\
\hline \multirow{3}{*}{2} & 0 & 8.60641 & 8.71642 & 8.40893 & 8.60958 & 8.04297 & 8.42849 \\
\hline & 1 & 6.98983 & 7.1038 & 6.74242 & 6.97228 & 6.27787 & 6.67923 \\
\hline & 2 & 6.01404 & 6.13386 & 5.72297 & 5.98105 & 5.16622 & 5.71731 \\
\hline \multirow{3}{*}{3} & 0 & 16.9869 & 17.5035 & 16.7959 & 17.3974 & 16.4414 & 17.2191 \\
\hline & 1 & 11.9593 & 11.4132 & 11.6773 & 11.2662 & 11.1543 & 11.017 \\
\hline & 2 & 9.70001 & 9.37428 & 9.34623 & 9.19483 & 8.68094 & 8.88769 \\
\hline
\end{tabular}

Taking $L_{a} / h=10, \tau=0.1, \zeta=0.1, \lambda=1, \quad \xi=1, p=1$, and $\Delta H=0,1 \%$, and $2 \%$, respectively, we reveal the variation of dimensionless first- and second-order vibration frequencies with temperature changing. It can be seen from Figures 4(a) and 4(b) that, the smaller the moisture concentration is, the greater the vibration frequency is. In addition, when the moisture concentration is fixed, the dimensionless vibration frequency decreases with the increase of temperature changing. For example, when the temperature changing increases from 0 to $200 \mathrm{~K}$ and the moisture concentration remains unchanged compared with $H_{0}$, the first-order vibration frequency decreases by $9.3 \%$ and the second-order decreases by $3.56 \%$. This is because the high temperature leads to expansion in nanoplate structures, which reduces the structural stiffness. Consequently, it is believed that the influence of temperature changing should be paid more attention to in lower-frequency vibration stage.

Subsequently, vibration frequencies versus continuous moisture concentrations are presented in Figures 5(a) and 5 (b). Let $\Delta T$ be $0,50 \mathrm{~K}$, and $100 \mathrm{~K}$, respectively, and other fixed parameters remain the same as Figures 4(a) and 4(b). The change of vibration frequencies with increasing moisture concentration can be observed. It can be revealed that the dimensionless vibration frequency is smaller when the temperature is higher. For example, if the temperature is kept as a constant at $T_{0}$, when the moisture concentration changes from 0 to $2 \%$, the first-order vibration frequency decreases by $12.25 \%$ and the second-order decreases by $4.63 \%$. The influence of hygrothermal environmental factors on fundamental frequencies of vibrating nanoplates is indispensable in the design of nanoplates working in special environments. The decrease of vibration frequency means that the equivalent stiffness of the nanoplate is weakened. Therefore, an increase in the moisture concentration may reduce the stiffness of functionally graded nanoplates. This is because the nanoplate structure degrades and swells with the adsorption of water molecules. Hence, in the actual design of NEMS, the damage effect of the high temperature and moisture on the structure should be considered.

In addition, the effect of the axial speed of functionally graded nanoplates on the vibration is considered. Let other parameters remain unchanged. For example, take $\Delta T=100 \mathrm{~K}, \Delta H=1 \%$, and $p=1$. Then, let $\tau=0.01, \zeta=0.05$; $\tau=0.05, \zeta=0.05$; and $\tau=0.05, \zeta=0.1$, respectively. The trend of the dimensionless vibration frequency with the increasing axial speed is presented in Figures 6(a) and 6(b). It can be seen that the vibration frequency increases when the nonlocal parameter becomes smaller or the material 


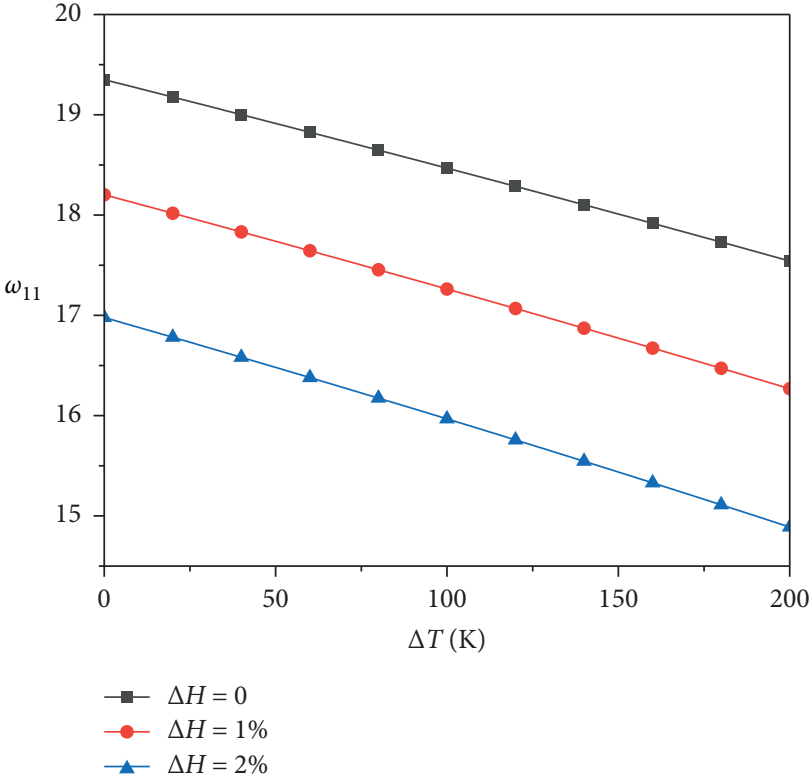

(a)

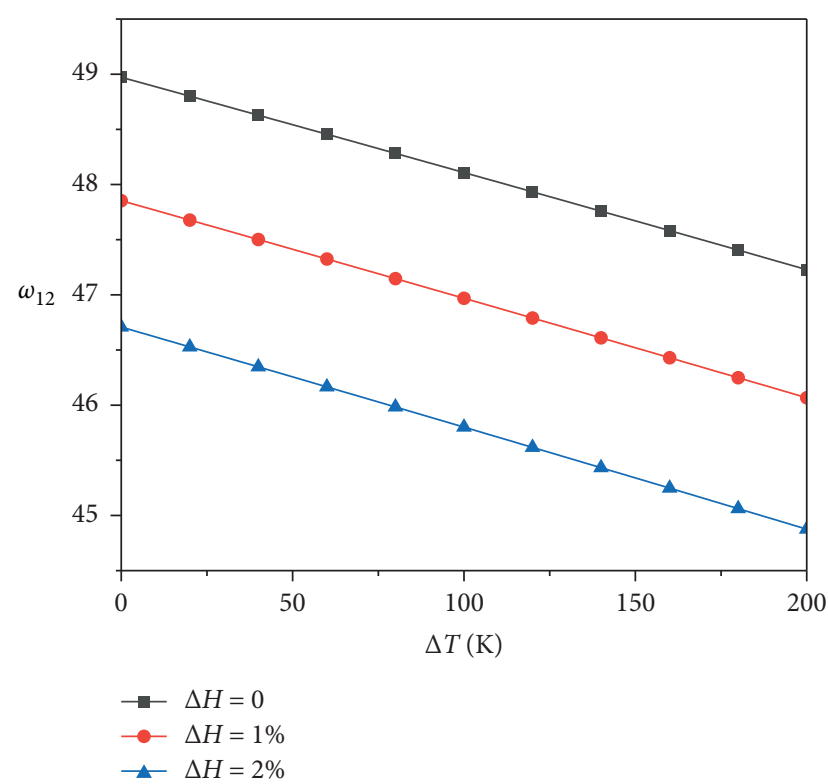

(b)

FIGURE 4: Variation of the vibration frequency with respect to temperature changing under different moisture concentrations. (a) The firstorder vibration frequency. (b) The second-order vibration frequency.

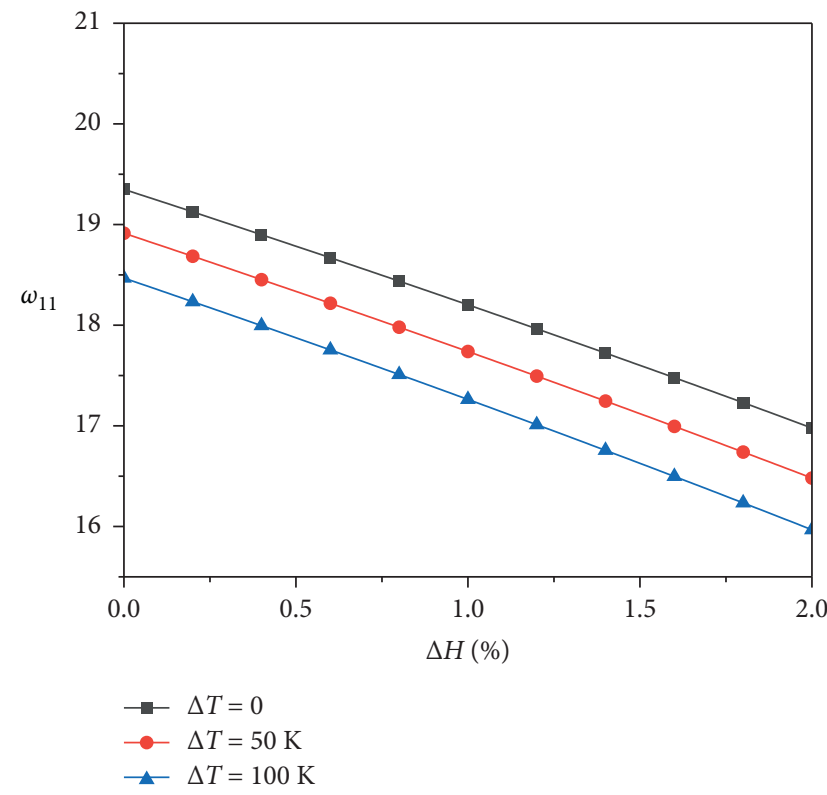

(a)

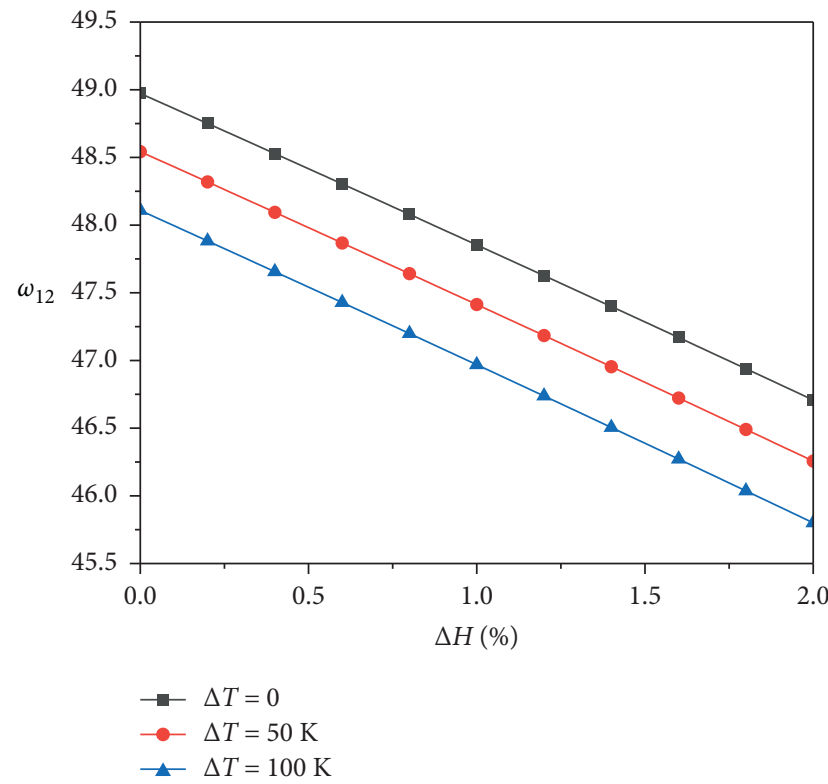

(b)

FIGURE 5: Variation of the vibration frequency with respect to moisture concentration under different temperature changing. (a) The firstorder vibration frequency. (b) The second-order vibration frequency.

characteristic scale parameter becomes larger. At the same time, it is apparent that with the increase of the axial speed, the vibration frequency decreases faster and faster. For example, choosing $\tau=0.05$ and $\zeta=0.05$, when the dimensionless speed increases from 0 to 2 , the first-order vibration frequency decreases by $9.36 \%$, while the dimensionless speed increases from 2 to 4 , the first-order frequency decreases by $59.99 \%$, and so the decline rate increases significantly.
Hence, the increase of axial speed leads to the instability of functionally graded nanoplates, and such a conclusion is a common sense which also holds at the macroscale. In the actual NEMS design, the appropriate speed should be chosen to ensure the stability of the system by considering the reduction effect of speed on the structure stiffness. Moreover, it can be observed that, when the nonlocal parameter is a constant, there is a significant difference between the 


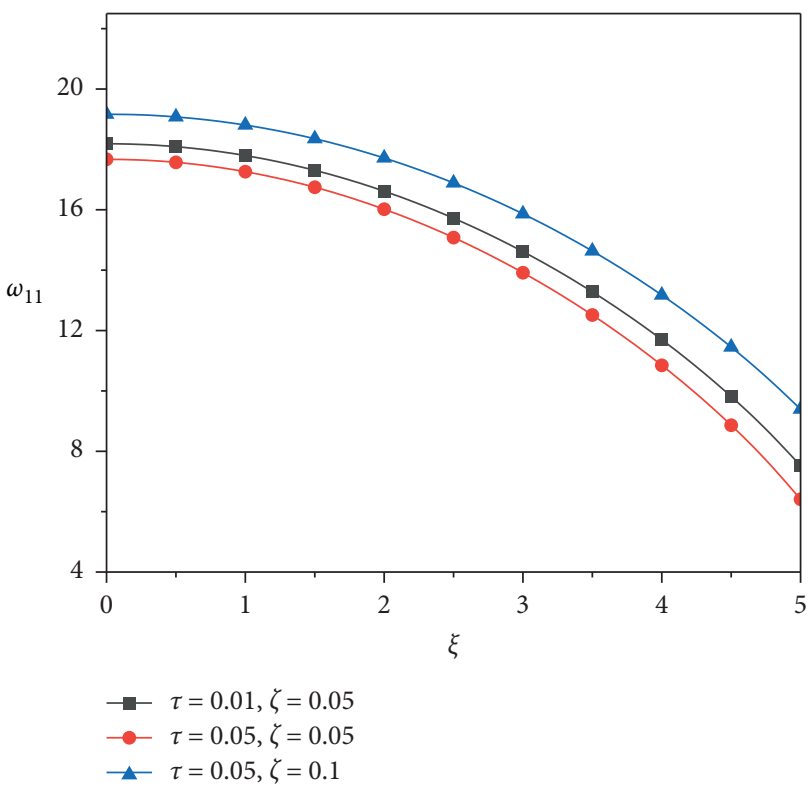

(a)

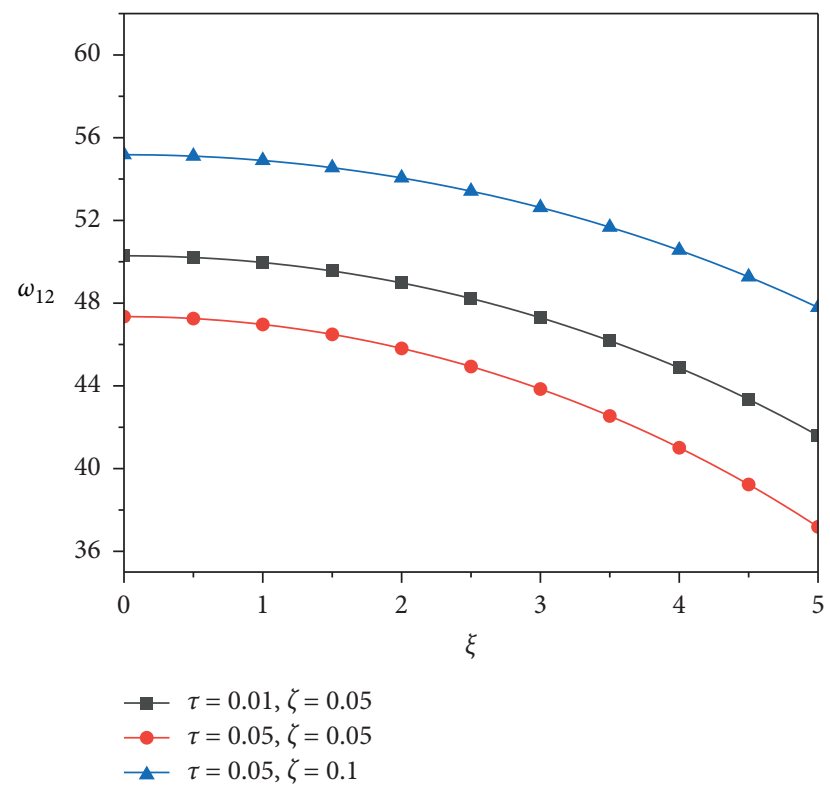

(b)

FiguRE 6: Variation of the vibration frequency with respect to the axial speed under different internal scale parameters. (a) The first-order vibration frequency. (b) The second-order vibration frequency.

vibration frequencies with $\zeta=0.05$ and $\zeta=0.1$. Therefore, there is a remarkable effect of the material characteristic scale parameter on the stability of the functionally graded nanoplates. Unlike the axial speed, the material characteristic scale parameter belongs to an internal influence factor, which is particularly significant at the nanoscale but can be ignored at the macroscale. It can also be seen from the Figure 6 that, for different nonlocal parameters and the material characteristic scale parameters, the curve has basically the same change trend. This shows that internal influence factors basically have no influence on the effect of axial speed changes on the system stability, which also indicates that the internal characteristic parameters affect little on the system at the macro scale.

As the gradient index increases, the properties of nanoplates gradually change from ceramic to metal. The effect of the gradient index on the dimensionless natural frequency under different material characteristic scale parameters is described in Figures 7(a) and 7(b). It can be seen that the natural frequency decreases as the gradient index increases, and for smaller material characteristic scale parameters, the rate of decrease is greater. In other words, when the material characteristic parameter is small, the change of the gradient index has a stronger influence on the vibration behaviors of the nanoplate. In the range of the gradient index from 0 to 4 , the vibration frequency is constant and the first-order frequency is always lower than the second-order frequency, indicating that the nanoplate system is stable in this range. Similarly, for both the first- and second-order frequencies, the frequencies are significantly different as the material characteristic scale parameters increase, which proves the size dependence of the nanoplates. For different $d$ material characteristic scale parameters, the slope of the curve in Figure 7 is different when it drops, which indicates that the material characteristic scale parameters will affect the stiffness effect of the gradient index on the system.

Then, the influence of the nonlocal parameter on the vibration stability is analyzed. The other parameters are kept unchanged and then take $\xi=1, \zeta=0.05 ; \xi=2.5$, $\zeta=0.05$; and $\xi=2.5, \zeta=0.1$, respectively. The changes of the dimensionless vibration frequency with increasing the nonlocal parameter under different dimensionless axial speeds and material characteristic scale parameters are plotted in Figures 8(a) and 8(b). It can be observed that the vibration frequency is larger with a smaller speed or a larger material characteristic scale parameter. Besides, it is clear that the dimensionless vibration frequency decreases with increasing the nonlocal parameter. Taking the case of $\xi=1$ and $\zeta=0.05$ as an example, when $\tau$ increases from 0 to 0.2 , the dimensionless first-order vibration frequency decreases by $34.99 \%$, and the secondorder decreases by $48.24 \%$, which reflects the softening behavior in the nonlocal effect. Meanwhile, we observe that, when $\xi=2.5$ and $\zeta=0.1$, the calculated results are in the middle in Figure $8(\mathrm{a})$, but they are at the top in Figure $8(\mathrm{~b})$. This is because the increase of stiffness caused by the increase of material characteristic scale parameter is greater than the stiffness weakening effect by the increase of the axial speed.

Furthermore, the influence of the nonlocal parameter on the vibration of functionally graded nanoplates with different rectangular shapes is discussed. The aspect ratio of the nanoplate $\lambda$ is chosen as $1,1.05$, and 1.1, respectively. As clearly visible in Figures 9(a) and 9(b), the dimensionless vibration frequency changes with the nonlocal parameter 


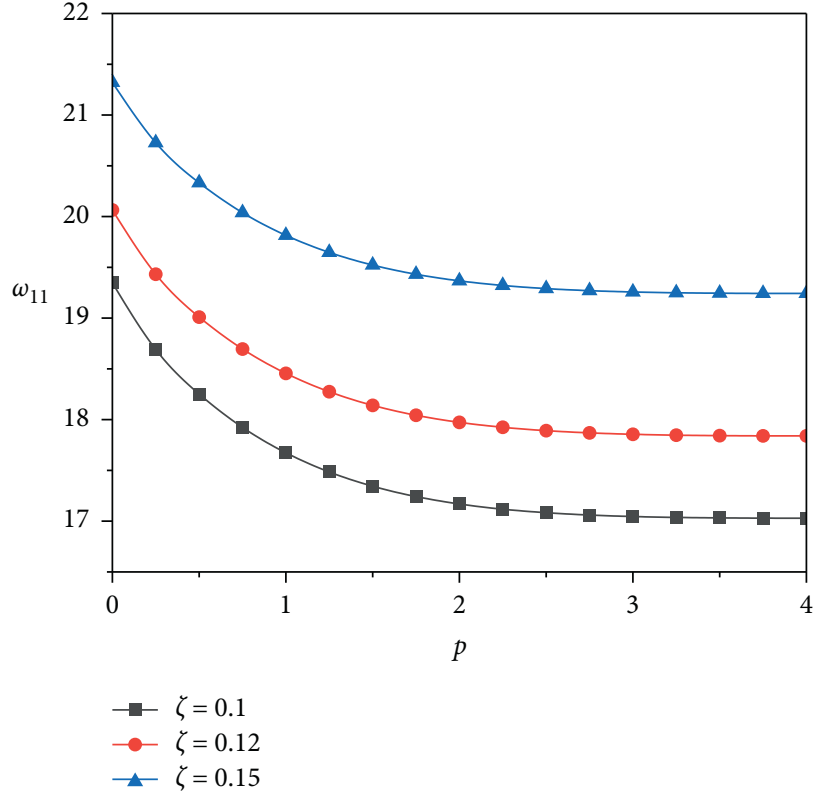

(a)

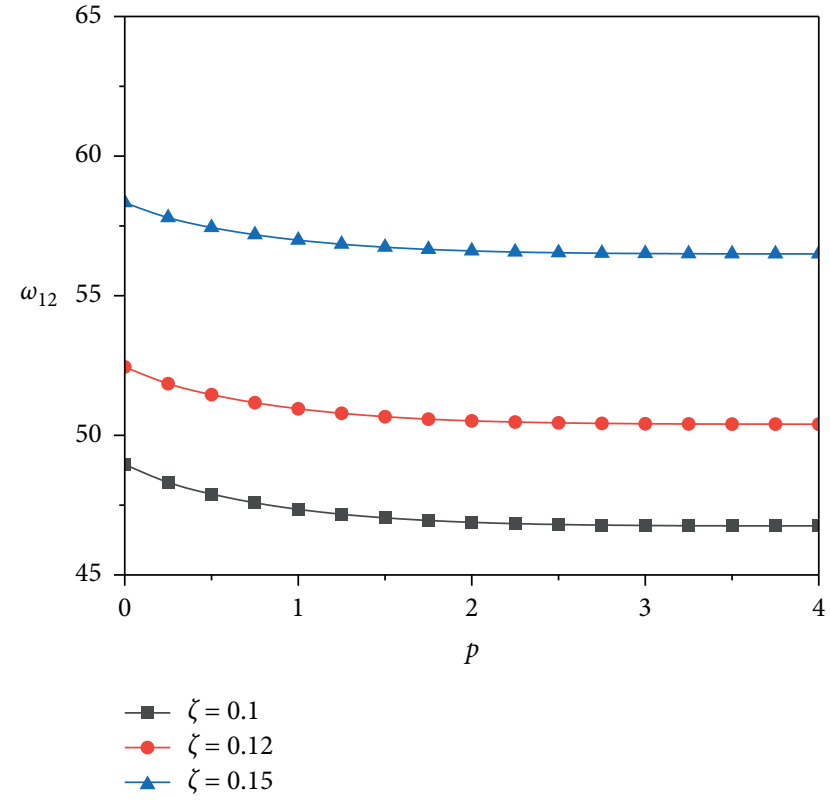

(b)

FIgURE 7: Variation of the vibration frequency with respect to the gradient index under different material characteristic scale parameters. (a) The first-order vibration frequency. (b) The second-order vibration frequency.

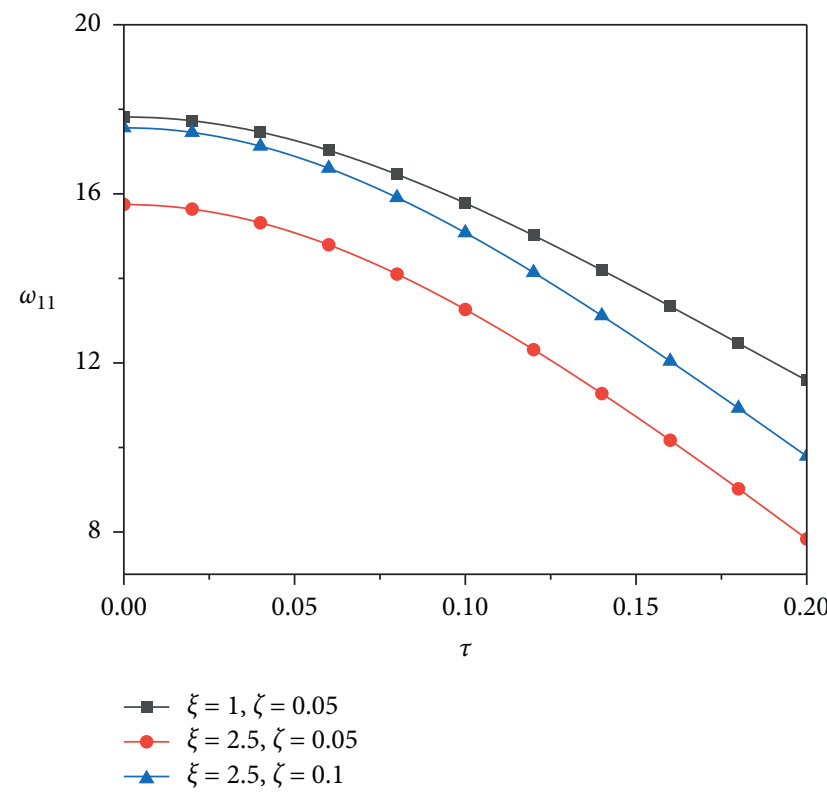

(a)

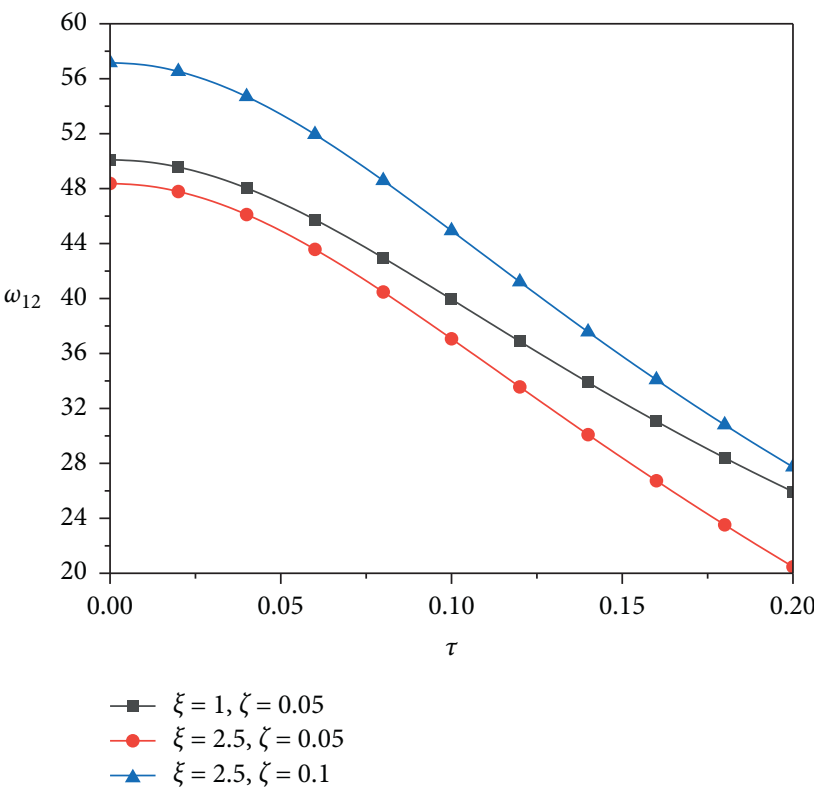

(b)

FIGURE 8: Effect of the nonlocal parameter on the vibration frequency of the functionally graded nanoplate: (a) the first-order vibration frequency and (b) the second-order vibration frequency.

under different aspect ratios. While the nonlocal parameter changes from 0 to 0.2 , if the aspect ratio is 1 , the first-order frequency decreases by $33.2 \%$ and the second-order frequency decreases by $46.26 \%$. If the aspect ratio is 1.05 , the first-order frequency decreases by $33.74 \%$ and the second- order frequency decreases by $46.58 \%$. If the aspect ratio is 1.1, the first-order frequency decreases by $34.29 \%$ and the second-order frequency decreases by $46.90 \%$. It is obvious that, in the case of higher-order vibration, when the aspect ratio increases, the vibration frequency decreases more and 


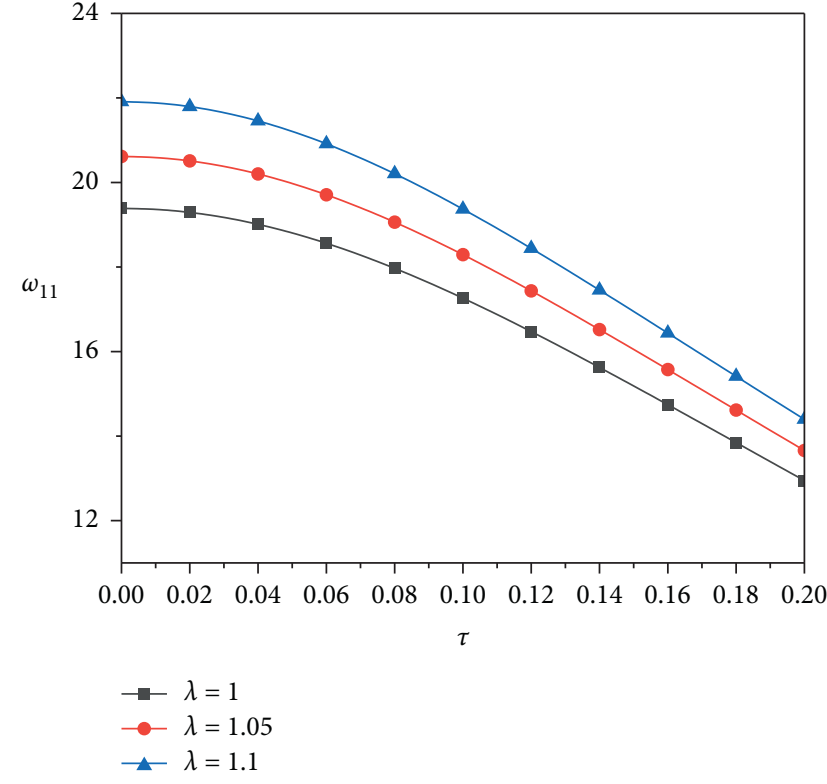

(a)

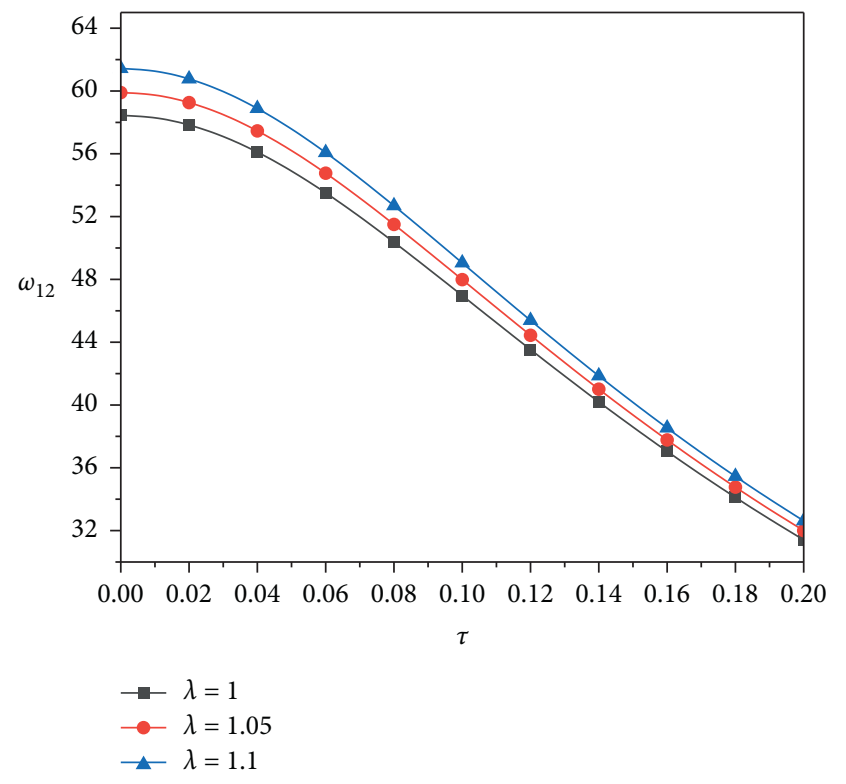

(b)

FIgURE 9: Variation of the vibration frequency with respect to the nonlocal parameter under different aspect ratios. (a) The first-order vibration frequency. (b) The second-order vibration frequency.

more significantly. The vibration of the nanoplate can be controlled by changing the shape of the nanoplate to avoid system resonance and ensure the stability of the structure.

Then, the influence of the material characteristic scale parameter is investigated. Keep the aspect ratio $\lambda=1$, and then select different axial speeds and nonlocal parameters as comparison; that is, taking $\xi=1, \tau=0.05 ; \xi=2, \tau=0.05$; and $\xi=2, \tau=0.1$, respectively. Figures 10 (a) and 10 (b) present the variation of vibration frequencies with the material characteristic scale parameter. Similar to previous conclusions, a lower axial speed and a smaller nonlocal parameter result in a higher vibration frequency. In addition, it can be seen that vibration frequencies increase with an increase in the material characteristic scale parameter. For instance, if $\xi=1$ and $\tau=0.05$, when the material characteristic scale parameter increases to 0.2 , then the first-order vibration frequency increases by $30.27 \%$ and the second-order increases by $79.42 \%$. This indicates that the stiffness of the nanoplate becomes larger while increasing the material characteristic scale parameter, which reflects the stiffness hardening effect. Besides, it is found that the influence of the material characteristic scale parameter on higher-order frequencies is greater than that on lower-order ones with different nonlocal parameters, reflecting a coupling effect between the material characteristic scale parameter and nonlocal parameter.

Finally, based on the coupling effect of the nonlocal parameter and the material characteristic scale parameter mentioned above, keeping $\xi=1$ and $\lambda=1$ invariant, the different material characteristic scale parameters are taken to observe the changes of vibration frequencies with the internal scale parameter ratio in Figure 11. It is concluded that vibration frequencies become greater in case of increasing the material characteristic scale parameter when the ratio $\tau / \zeta$ is less than 1 (i.e., $\tau<\zeta$ ). Herein, the material characteristic scale parameter representing the strain gradient effect plays a dominant role, and the increase of vibration frequencies means the stiffness hardening. On the other hand, vibration frequencies diminish with an increase in the material characteristic scale parameter in the case of $\tau>\zeta$. Herein, the nonlocal parameter representing the nonlocal effect dominates the mechanism of stiffness variation and thus exhibits a softening behavior. In particular, the vibration frequency is restored to that of the classical thin plate vibration theory when the ratio $\tau / \zeta$ is equal to 1 (i.e., $\tau=\zeta$ ). The stiffness softening and hardening behaviors were commonly seen in the nanostructures. In fact, the coupling effect of the nonlocal parameter and material characteristic scale parameter results in the behaviors of stiffness softening and hardening. In the nonlocal strain gradient theory, two nanoscaled internal scale parameters including the nonlocal parameter and the material characteristic scale parameter are in the same magnitude, and the nonlocal effect and strain gradient effect respectively represented by the two parameters are equivalent. Moreover, the two kinds of internal scale effects are coupled in out-of-plane vibrations of axially moving functionally graded nanoplates in hygrothermal environments as analyzed before. In this coupling interaction, the effect of a nanoscaled nonlocal parameter is almost equal to that of the nanoscaled material characteristic scale parameter. This explains why the boundary between the softening behavior and hardening behavior is determined by the magnitude relationship between the two internal parameters.

In this study, the competition relationship between different internal characteristic parameters under the 


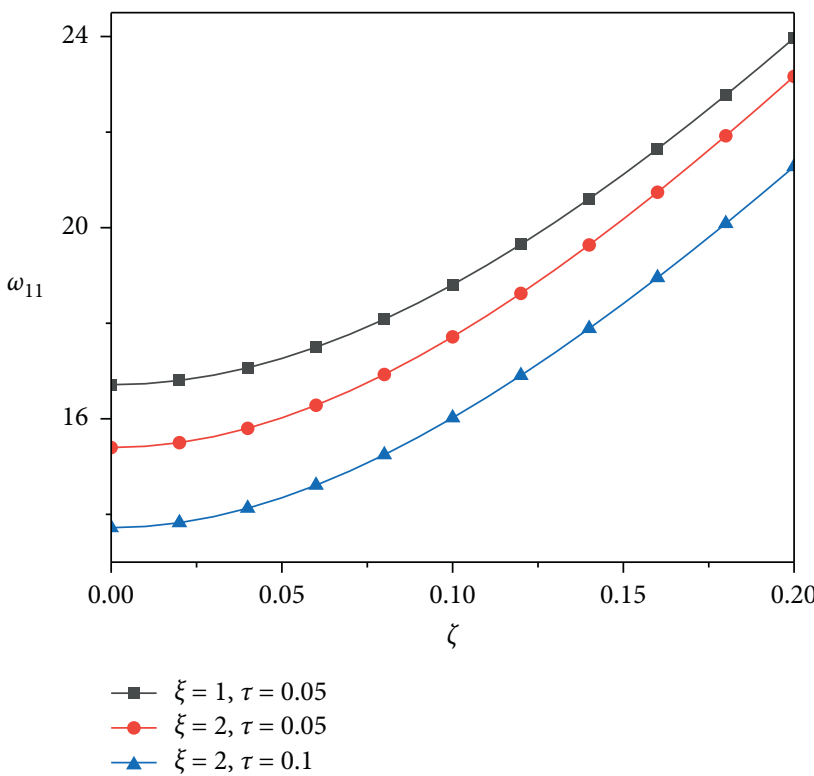

(a)

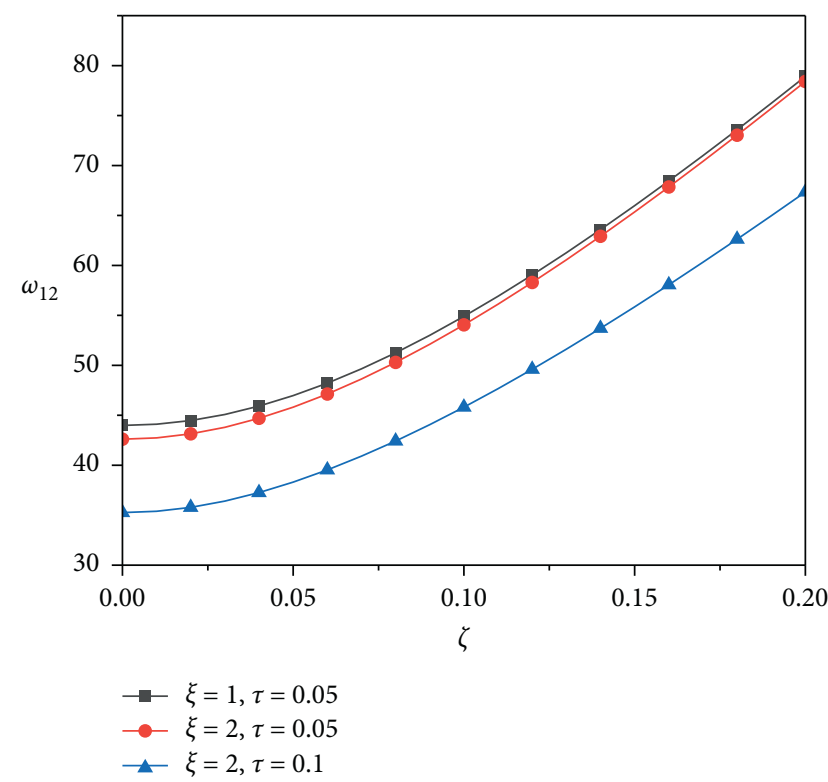

(b)

FIgURE 10: Variation of the vibration frequency with respect to the material characteristic scale parameter. (a) The first-order vibration frequency. (b) The second-order vibration frequency.

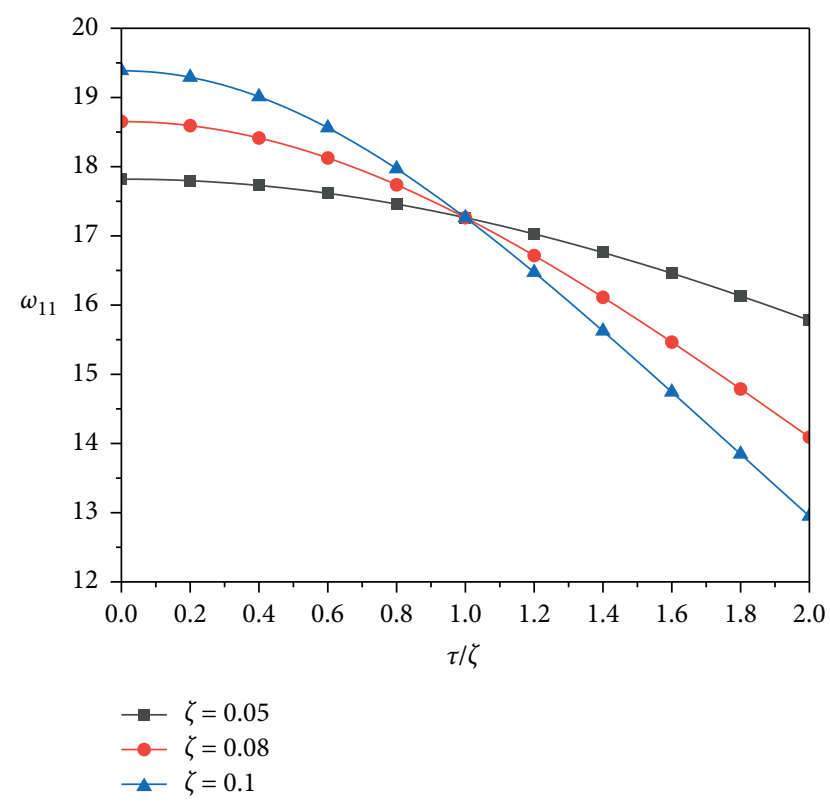

FIGURE 11: Variation of the first-order vibration frequency with respect to the ratio of the nonlocal parameter to the material characteristic scale parameter.

external elastic deformation and rigid motion is revealed for the first time, which can provide a reference for the design of MEMS/NEMS with a macroscopic motion in hygrothermal environments. Due to the coupling effect, the nonlocal effect and strain gradient effect should be considered comprehensively to control the stability of the system in an actual situation. It should also be mentioned that although the coupling phenomenon in the nonlocal and strain gradient effects is observed, the influences of internal characteristic parameters and external physical parameters on axially moving hygrothermal functionally graded nanoplates are independent.

\section{Conclusions}

Based on the nonlocal strain gradient theory, the functionally graded nanoplate with an axial motion in hygrothermal environments is investigated. The governing equation of motion for a vibrating and moving functionally graded nanoplate is derived via Hamilton's principle. The out-of-plane vibration characteristics of functionally graded nanoplates with boundary constraints simply supported on four sides are presented by solving the frequency-domain equation numerically. The effects of the dimensionless parameters on the vibration frequencies such as the temperature changing, moisture concentration, axial speed, gradient index, nonlocal parameter, material characteristic scale parameter, and aspect ratio are discussed. The following conclusions are obtained:

(i) The effect of hygrothermal environmental factors on fundamental frequencies of vibrating nanoplates is obvious and the increase of moisture concentration and temperature change reduces the vibration frequency.

(ii) The variation of axial speed plays an important role in the stability of axially moving nanoplates. With the increase of axial speed, the vibration frequency decreases faster and faster. Hence, the increase of speed leads to the instability of the system. 
(iii) The shape of the nanoplate and the mixing mode of its internal components have effects on the dynamic stability of the system. The greater the aspect ratio is, the higher the vibration frequency is. When the gradient index becomes larger, the natural frequency gets decreasing.

(iv) The vibration frequencies decrease with the increase of the nonlocal parameter and increase with the increase of the material characteristic scale parameter. The two internal characteristic parameters influence and restrict each other. If the nonlocal parameter is greater than the material characteristic scale parameter, the nonlocal effect plays a dominant role, and the stiffness softening is observed. If not, the strain gradient effect dominates, and the stiffness hardening is observed. Hence, the competition relationship existing in internal characteristic parameters at the micro/nanoscale is revealed.

\section{Data Availability}

The data generated or analyzed during this study are included within the article.

\section{Conflicts of Interest}

The authors declare that there are no conflicts of interest.

\section{Authors' Contributions}

Chengxiu Zhu and Jianwei Yan contributed equally to this work.

\section{Acknowledgments}

This work was supported by the https://doi.org/10.13039/ 501100001809 National Natural Science Foundation of China (Nos. 11972240 and 12072112), National Natural Science Foundation Excellent Youth Cultivation Project (No. 20202ZDB01001), and Guangxi Key Laboratory of Cryptography and Information Security (No. GCIS201905).

\section{References}

[1] M. Hadj Said, F. Tounsi, S. Surya, B. Mezghani, M. Masmoudi, and V. Rao, "A MEMS-based shifted membrane electrodynamic microsensor for microphone applications," Journal of Vibration and Control, vol. 24, no. 1, pp. 208-222, 2018.

[2] H. L. Cao, Y. J. Zhang, C. Shen, Y. Liu, and X. Wang, "Temperature energy influence compensation for MEMS vibration gyroscope based on rbf NN-GA-KF method," Shock and Vibration, vol. 2018, Article ID 2830686, 2018.

[3] G. Li, B. Yin, L. W. Zhang, and K. M. Liew, "Modeling microfracture evolution in heterogeneous composites via a coupled cohesive phase-field model," Journal of the Mechanics and Physics of Solids, vol. 142, Article ID 103968, 2020.

[4] L. Z. Xu, Y. L. Liu, and X. R. Fu, "Effects of the van der Waals force on the dynamics performance for a micro resonant pressure sensor," Shock and Vibration, vol. 2016, Article ID 3426196, 11 pages, 2016.
[5] L. F. Wang and H. Y. Hu, "Flexural wave propagation in single-walled carbon nanotubes," Physical Review B, vol. 71, no. 19, Article ID 195412, 2005.

[6] B. Akgöz and O. Civalek, "Strain gradient elasticity and modified couple stress models for buckling analysis of axially loaded micro-scaled beams," International Journal of Engineering Science, vol. 49, no. 11, pp. 1268-1280, 2011.

[7] Y.-W. Zhang, L. Zhou, B. Fang, and T.-Z. Yang, "Quantum effects on thermal vibration of single-walled carbon nanotubes conveying fluid," Acta Mechanica Solida Sinica, vol. 30, no. 5, pp. 550-556, 2017.

[8] X. Yang and C. W. Lim, "Nonlinear vibrations of nano-beams accounting for nonlocal effect using a multiple scale method," Science in China-Series E: Technological Sciences, vol. 52, no. 3, pp. 617-621, 2009.

[9] W. P. Hu, Y. L. Huai, M. B. Xu et al., "Mechanoelectrical flexible hub-beam model of ionic-type solvent-free nanofluids," Mechanical Systems and Signal Processing, vol. 159, Article ID 107833, 2021.

[10] W.-M. Zhang and G. Meng, "Nonlinear dynamic analysis of electrostatically actuated resonant MEMS sensors under parametric excitation," IEEE Sensors Journal, vol. 7, no. 3-4, pp. 370-380, 2007.

[11] W.-M. Zhang, G. Meng, and K.-X. Wei, "Dynamics of nonlinear coupled electrostatic micromechanical resonators under two-frequency parametric and external excitations," Shock and Vibration, vol. 17, no. 6, pp. 759-770, 2010.

[12] A. Darabi and A. R. Vosoughi, "A hybrid inverse method for small scale parameter estimation of FG nanobeams," Steel and Composite Structures, vol. 20, no. 5, pp. 1119-1131, 2016.

[13] L. Librescu, S. Y. Oh, and O. Song, "Thin-walled beams made of functionally graded materials and operating in a high temperature environment: vibration and stability," Journal of Thermal Stresses, vol. 28, no. 6-7, pp. 649-712, 2005.

[14] V. Birman and L. W. Byrd, "Modeling and analysis of functionally graded materials and structures," Applied Mechanics Reviews, vol. 60, no. 1-6, pp. 195-216, 2007.

[15] Z. Wang, X. Shi, H. Huang et al., "Magnetically actuated functional gradient nanocomposites for strong and ultradurable biomimetic interfaces/surfaces," Materials Horizons, vol. 4, no. 5, pp. 869-877, 2017.

[16] M. M. Ameen, K. Prabhul, G. Sivakumar, P. P. Abraham, U. B. Jayadeep, and C. B. Sobhan, "Molecular dynamics modeling of latent heat enhancement in nanofluids," International Journal of Thermophysics, vol. 31, no. 6, pp. 1131$1144,2010$.

[17] A. Hemmasizadeh, M. Mahzoon, E. Hadi, and R. Khandan, “A method for developing the equivalent continuum model of a single layer graphene sheet," Thin Solid Films, vol. 516, no. 21, pp. 7636-7640, 2008.

[18] J. D. Lee, X. Wang, and Y. Chen, "Multiscale computation for nano/micromaterials," Journal of Engineering Mechanics, vol. 135, no. 3, pp. 192-202, 2009.

[19] G. Huetter, "On the micro-macro relation for the microdeformation in the homogenization towards micromorphic and micropolar continua," Journal of the Mechanics and Physics of Solids, vol. 127, pp. 62-79, 2019.

[20] R. A. Toupin, "Theories of elasticity with couple-stress," Archive for Rational Mechanics and Analysis, vol. 17, no. 2, pp. 85-112, 1964.

[21] R. D. Mindlin and N. N. Eshel, "On first strain-gradient theories in linear elasticity," International Journal of Solids and Structures, vol. 4, no. 1, pp. 109-124, 1968. 
[22] A. C. Eringen and D. G. B. Edelen, "On nonlocal elasticity," International Journal of Engineering Science, vol. 10, no. 3, pp. 233-248, 1972.

[23] L. Li, R. Lin, and T. Ng, "Contribution of nonlocality to surface elasticity," International Journal of Engineering Science, vol. 152, Article ID 103311, 2020.

[24] L. Li, R. Lin, and Y. Hu, "Cross-section effect on mechanics of nonlocal beams," Archive of Applied Mechanics, vol. 91, no. 4, pp. 1541-1556, 2021.

[25] M. H. Jalaei and Ö. Civalek, "On dynamic instability of magnetically embedded viscoelastic porous FG nanobeam," International Journal of Engineering Science, vol. 143, pp. 14-32, 2019.

[26] L. Lu, G.-L. She, and X. Guo, "Size-dependent postbuckling analysis of graphene reinforced composite microtubes with geometrical imperfection," International Journal of $\mathrm{Me}$ chanical Sciences, vol. 199, Article ID 106428, 2021.

[27] N. Challamel and C. M. Wang, "The small length scale effect for a non-local cantilever beam: a paradox solved," Nanotechnology, vol. 19, no. 34, Article ID 345703, 2008.

[28] C. W. Lim, G. Zhang, and J. N. Reddy, "A higher-order nonlocal elasticity and strain gradient theory and its applications in wave propagation," Journal of the Mechanics and Physics of Solids, vol. 78, pp. 298-313, 2015.

[29] S. Fallahzadeh Rastehkenari, "Random vibrations of functionally graded nanobeams based on unified nonlocal strain gradient theory," Microsystem Technologies, vol. 25, no. 2, pp. 691-704, 2019.

[30] J. Wang and H. Shen, "Nonlinear vibrations of axially moving simply supported viscoelastic nanobeams based on nonlocal strain gradient theory," Journal of Physics. Condensed Matter: An Institute of Physics Journal, vol. 31, no. 48, Article ID 485403, 2019.

[31] P. R. Saffari, M. Fakhraie, and M. A. Roudbari, "Nonlinear vibration of fluid conveying cantilever nanotube resting on visco-pasternak foundation using non-local strain gradient theory," Micro \& Nano Letters, vol. 15, no. 3, pp. 181-186, 2020.

[32] L. Li, X. Li, and Y. Hu, "Free vibration analysis of nonlocal strain gradient beams made of functionally graded material," International Journal of Engineering Science, vol. 102, pp. 77-92, 2016.

[33] L. Lu, S. Wang, M. Li, and X. Guo, "Free vibration and dynamic stability of functionally graded composite microtubes reinforced with graphene platelets," Composite Structures, vol. 272, Article ID 114231, 2021.

[34] L. Lu, L. Zhu, X. Guo, J. Zhao, and G. Liu, "A nonlocal strain gradient shell model incorporating surface effects for vibration analysis of functionally graded cylindrical nanoshells," Applied Mathematics and Mechanics, vol. 40, no. 12, pp. 1695-1722, 2019.

[35] V. Pham Van and A. Tounsi, "The role of spatial variation of the nonlocal parameter on the free vibration of functionally graded sandwich nanoplates," Engineering with Computers, 2021.

[36] H. Ding, M. Zhu, and L. Chen, "Dynamic stiffness method for free vibration of an axially moving beam with generalized boundary conditions," Applied Mathematics and Mechanics, vol. 40, no. 7, pp. 911-924, 2019.

[37] S. Refrafi, A. A. Bousahla, A. Bouhadra et al., "Effects of hygrothermo-mechanical conditions on the buckling of FG sandwich plates resting on elastic foundations," Computers and Concrete, vol. 25, no. 4, pp. 311-325, 2020.
[38] A. Tounsi, S. U. Al-Dulaijan, M. A. Al-Osta et al., "A four variable trigonometric integral plate theory for hygrothermo-mechanical bending analysis of AFG ceramic-metal plates resting on a two-parameter elastic foundation," Steel and Composite Structures, vol. 34, no. 4, pp. 511-524, 2020.

[39] J. Duan, D. Zhang, and W. Wang, "Flutter and divergence instability of axially-moving nanoplates resting on a viscoelastic foundation," Applied Sciences-Basel, vol. 9, no. 6, Article ID 1097, 2019.

[40] J. J. Liu, C. Li, X. L. Fan, and L. H. Tong, "Transverse free vibration and stability of axially moving nanoplates based on nonlocal elasticity theory," Applied Mathematical Modelling, vol. 45, pp. 65-84, 2017.

[41] C. Li, P. Y. Wang, Q. Y. Luo, and S. Li, "Free vibration of axially moving functionally graded nanoplates based on the nonlocal strain gradient theory," International Journal of Acoustics and Vibration, vol. 25, no. 4, pp. 587-596, 2020.

[42] M. Esmaeilzadeh and M. Kadkhodayan, "Numerical investigation into dynamic behaviors of axially moving functionally graded porous sandwich nanoplates reinforced with graphene platelets," Materials Research Express, vol. 6, no. 10, Article ID 1050b7, 2019.

[43] J. N. Reddy, "Analysis of functionally graded plates," International Journal for Numerical Methods in Engineering, vol. 47, no. 1-3, pp. 663-684, 2000.

[44] S. Blooriyan, R. Ansari, A. Darvizeh, R. Gholami, and H. Rouhi, "Pre- and post-buckling analysis of FG cylindrical nanoshells in thermal environment considering the surface stress effect," Materials Research Express, vol. 6, no. 9, Article ID 095067, 2019.

[45] D.-G. Zhang and Y.-H. Zhou, "A theoretical analysis of FGM thin plates based on physical neutral surface," Computational Materials Science, vol. 44, no. 2, pp. 716-720, 2008.

[46] M. Alshaqaq and M. A. Hawwa, "Nonlinear behavior of a vibrating axially moving small-size beam under an electrostatic force," Zamm-Zeitschrift Fur Angewandte Mathematik Und Mechanik, vol. 100, no. 9, Article ID e201900104, 2020.

[47] Y.-Q. Tang and L.-Q. Chen, "Stability analysis and numerical confirmation in parametric resonance of axially moving viscoelastic plates with time-dependent speed," European Journal of Mechanics-A: Solids, vol. 37, pp. 106-121, 2013.

[48] S. Natarajan, S. Chakraborty, M. Thangavel, S. Bordas, and T. Rabczuk, "Size-dependent free flexural vibration behavior of functionally graded nanoplates," Computational Materials Science, vol. 65, pp. 74-80, 2012.

[49] A. A. Jandaghian and O. Rahmani, "Vibration analysis of functionally graded piezoelectric nanoscale plates by nonlocal elasticity theory: an analytical solution," Superlattices and Microstructures, vol. 100, pp. 57-75, 2016.

[50] M. R. Barati and H. Shahverdi, "An analytical solution for thermal vibration of compositionally graded nanoplates with arbitrary boundary conditions based on physical neutral surface position," Mechanics of Advanced Materials and Structures, vol. 24, no. 10, pp. 840-853, 2017.

[51] H. Shahverdi and M. R. Barati, "Vibration analysis of porous functionally graded nanoplates," International Journal of Engineering Science, vol. 120, pp. 82-99, 2017.

[52] Q. Wang and C. M. Wang, "The constitutive relation and small scale parameter of nonlocal continuum mechanics for modelling carbon nanotubes," Nanotechnology, vol. 18, no. 7, Article ID 075702, 2007.

[53] C. Li, S. K. Lai, and X. Yang, "On the nano-structural dependence of nonlocal dynamics and its relationship to the 
upper limit of nonlocal scale parameter," Applied Mathematical Modelling, vol. 69, pp. 127-141, 2019.

[54] L. Lu, X. Guo, and J. Zhao, "A unified size-dependent plate model based on nonlocal strain gradient theory including surface effects," Applied Mathematical Modelling, vol. 68, pp. 583-602, 2019. 